\title{
On the dislocation density tensor in the Cosserat theory of elastic shells
}

\author{
Mircea Bîrsan ${ }^{1}$ and Patrizio Neff ${ }^{2}$
}

September 19, 2018

\begin{abstract}
We consider the Cosserat continuum in its finite strain setting and discuss the dislocation density tensor as a possible alternative curvature strain measure in three-dimensional Cosserat models and in Cosserat shell models. We establish a close relationship (one-to-one correspondence) between the new shell dislocation density tensor and the bending-curvature tensor of 6-parameter shells.
\end{abstract}

\section{Indroduction}

The Cosserat type theories have recently seen a tremendous renewed interest for their prospective applicability to model physical effects beyond the classical ones. These comprise notably the so-called size-effects ("smaller is stiffer").

In a finite strain Cosserat-type framework the group of proper rotations $\mathrm{SO}(3)$ has a dominant place. The original idea of the Cosserat brothers [16 to consider independent rotational degrees of freedom in addition to the macroscopic displacement was heavily motivated by their treatment of plate and shell theory. Indeed, in shell theory it is natural to attach a preferred orthogonal frame (triad) at any point of the surface, one vector of which is the normal to the midsurface, the other two vectors lying in the tangent plane. This is the notion of the "trièdre caché". The idea to consider then an orthogonal frame which is not strictly linked to the surface, but constitutively coupled, leads to the notion of the "trièdre mobile". And this then is already giving rise to a prototype Cosserat shell (6-parameter) theory. For an insightful review of various Cosserat-type shell models we refer to 8 .

However, the Cosserat brothers have never proposed any more specific constitutive framework, apart from postulating euclidean invariance (frame-indifference) and hyperelasticity. For specific problems it is necessary to choose a constitutive framework and to determine certain strain and curvature measures. This task is still not conclusively done, see e.g. [30.

Among the existing models for Cosserat-type shells we mention the theory of simple elastic shells [7, which has been developed by [34, 35] and [5, 6]. Later, this theory has been successfully applied to describe the mechanical behavior of laminated, functionally graded, viscoelastic or porous plates in [1, 2, 3, 4, and of multi-layered, orthotropic, thermoelastic shells in 10, 11, 14, 31. Another remarkable approach is the general 6-parameter theory of elastic shells presented in [23, 15, 18. Although the starting point is different, one can see

\footnotetext{
${ }^{1}$ Lehrstuhl für Nichtlineare Analysis und Modellierung, Fakultät für Mathematik, Universität Duisburg-Essen, Thea-Leymann Str. 9, 45127 Essen, Germany; and Alexandru Ioan Cuza University of Iaşi, Department of Mathematics, Blvd. Carol I, no. 11, 700506 Iaşi, Romania, mircea.birsan@uni-due.de

${ }^{2} \mathrm{Head}$ of Lehrstuhl für Nichtlineare Analysis und Modellierung, Fakultät für Mathematik, Universität Duisburg-Essen, Thea-Leymann Str. 9, 45127 Essen, Germany patrizio.neff@uni-due.de
} 
that the kinematical structure of the nonlinear 6-parameter shell theory is identical to that of a Cosserat shell model, see also [12, 13.

In this paper we would like to draw attention to alternative curvature measures, motivated by dislocation theory, which can also profitably be used in the three-dimensional Cosserat model and the Cosserat shell model. The object of interest is Nye's dislocation density tensor Curl $\boldsymbol{P}$. Within the restriction to proper rotations it turns out that Nye's tensor provides a complete control of all spatial derivatives of rotations 28 and we rederive this property for micropolar continua using general curvilinear coordinates. Then we focus on shell-curvature measures and define a new shell dislocation density tensor using the surface Curl operator. Then, we prove that a relation analogous to Nye's formula holds also for Cosserat (6-parameter) shells.

The paper is structured as follows. In Section 2 we present the kinematics of a threedimensional Cosserat continuum, as well as the appropriate strain measures and curvature strain measures, written in curvilinear coordinates. Here, we show the close relationship between the wryness tensor and the dislocation density tensor, including the corresponding Nye's formula. In Section 3 we define the Curl operator on surfaces and present several representations using surface curvilinear coordinates. These relations are then used in Section 4 to introduce the new shell dislocation density tensor and to investigate its relationship to the elastic shell bending-curvature tensor of 6-parameter shells.

\section{Strain measures of a three-dimensional Cosserat model in curvilinear coordinates}

Let $\mathcal{B}$ be a Cosserat elastic body which occupies in its reference (initial) configuration the domain $\Omega_{\xi} \subset \mathbb{R}^{3}$. A generic point of $\Omega_{\xi}$ will be denoted by $\left(\xi_{1}, \xi_{2}, \xi_{3}\right)$. The deformation of the Cosserat body is described by a vectorial map $\varphi_{\xi}$ and a microrotation tensor $\boldsymbol{R}_{\xi}$,

$$
\boldsymbol{\varphi}_{\xi}: \Omega_{\xi} \rightarrow \Omega_{c}, \quad \boldsymbol{R}_{\xi}: \Omega_{\xi} \rightarrow \mathrm{SO}(3)
$$

where $\Omega_{c}$ is the deformed (current) configuration. Let $\left(x_{1}, x_{2}, x_{3}\right)$ be some general curvilinear coordinates system on $\Omega_{\xi}$. Thus, we have a parametric representation $\Theta$ of the domain $\Omega_{\xi}$

$$
\boldsymbol{\Theta}: \Omega \rightarrow \Omega_{\xi}, \quad \boldsymbol{\Theta}\left(x_{1}, x_{2}, x_{3}\right)=\left(\xi_{1}, \xi_{2}, \xi_{3}\right),
$$

where $\Omega \subset \mathbb{R}^{3}$ is a bounded domain with Lipschitz boundary $\partial \Omega$. The covariant base vectors with respect to these curvilinear coordinates are denoted by $\boldsymbol{g}_{i}$ and the contravariant base vectors by $\boldsymbol{g}^{j}(i, j=1,2,3)$, i.e.

$$
\boldsymbol{g}_{i}=\frac{\partial \boldsymbol{\Theta}}{\partial x_{i}}=\boldsymbol{\Theta}_{, i}, \quad \boldsymbol{g}^{j} \cdot \boldsymbol{g}_{i}=\delta_{i}^{j}
$$

where $\delta_{i}^{j}$ is the Kronecker symbol. We employ the usual conventions for indices: the Latin indices $i, j, k, \ldots$ range over the set $\{1,2,3\}$, while the Greek indices $\alpha, \beta, \gamma, \ldots$ are confined to the range $\{1,2\}$; the comma preceding an index $i$ denotes partial derivatives with respect to $x_{i}$; the Einstein summation convention over repeated indices is also used.

Introducing the deformation function $\varphi$ by the composition

$$
\boldsymbol{\varphi}:=\boldsymbol{\varphi}_{\xi} \circ \boldsymbol{\Theta}: \Omega \rightarrow \Omega_{c}, \quad \boldsymbol{\varphi}\left(x_{1}, x_{2}, x_{3}\right):=\boldsymbol{\varphi}_{\xi}\left(\boldsymbol{\Theta}\left(x_{1}, x_{2}, x_{3}\right)\right),
$$

we can express the (elastic) deformation gradient $\boldsymbol{F}$ as follows

$$
\boldsymbol{F}:=\nabla_{\xi} \boldsymbol{\varphi}_{\xi}\left(\xi_{1}, \xi_{2}, \xi_{3}\right)=\nabla_{x} \boldsymbol{\varphi}\left(x_{1}, x_{2}, x_{3}\right) \cdot\left[\nabla_{x} \boldsymbol{\Theta}\left(x_{1}, x_{2}, x_{3}\right)\right]^{-1} .
$$




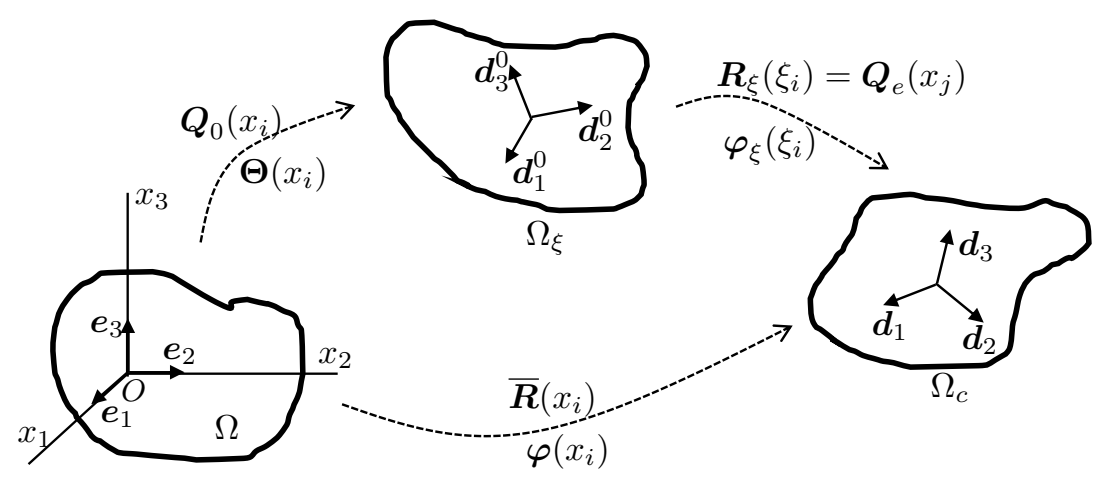

Figure 1: The reference (initial) configuration $\Omega_{\xi}$ of the Cosserat continuum, the deformed (current) configuration $\Omega_{c}$ and the parameter domain $\Omega$ of the curvilinear coordinates $\left(x_{1}, x_{2}, x_{3}\right)$. The triads of directors $\left\{\boldsymbol{d}_{i}\right\}$ and $\left\{\boldsymbol{d}_{i}^{0}\right\}$ satisfy the relations $\boldsymbol{d}_{i}=\boldsymbol{Q}_{e} \boldsymbol{d}_{i}^{0}=\overline{\boldsymbol{R}} \boldsymbol{e}_{i}$ and $\boldsymbol{d}_{i}^{0}=\boldsymbol{Q}_{0} \boldsymbol{e}_{i}$, where $\boldsymbol{Q}_{e}$ is the elastic microrotation field, $\boldsymbol{Q}_{0}$ the initial microrotation, and $\overline{\boldsymbol{R}}$ the total microrotation field.

Using the direct tensor notation, we can write

$$
\nabla_{x} \boldsymbol{\varphi}=\boldsymbol{\varphi}_{, i} \otimes \boldsymbol{e}_{i}, \quad \nabla_{x} \boldsymbol{\Theta}=\boldsymbol{g}_{i} \otimes \boldsymbol{e}_{i}, \quad\left[\nabla_{x} \boldsymbol{\Theta}\right]^{-1}=\boldsymbol{e}_{j} \otimes \boldsymbol{g}^{j},
$$

where $\boldsymbol{e}_{i}$ are the unit vectors along the coordinate axes $O x_{i}$ in the parameter domain $\Omega$. Then, the deformation gradient can be expressed by

$$
\boldsymbol{F}=\varphi_{, i} \otimes \boldsymbol{g}^{i}
$$

The orientation and rotation of points in Cosserat (micropolar) media can also be described by means of triads of orthonormal vectors (called directors) attached to every point. We denote by $\left\{\boldsymbol{d}_{i}^{0}\right\}$ the triad of directors $(i=1,2,3)$ in the reference configuration $\Omega_{\xi}$ and by $\left\{\boldsymbol{d}_{i}\right\}$ the directors in the deformed configuration $\Omega_{c}$, see Figure 1 . We introduce the elastic microrotation $\boldsymbol{Q}_{e}$ as the composition

$$
\boldsymbol{Q}_{e}:=\boldsymbol{R}_{\xi} \circ \boldsymbol{\Theta}: \Omega \rightarrow \mathrm{SO}(3), \quad \boldsymbol{Q}_{e}\left(x_{1}, x_{2}, x_{3}\right):=\boldsymbol{R}_{\xi}\left(\boldsymbol{\Theta}\left(x_{1}, x_{2}, x_{3}\right)\right),
$$

which can be characterized with the help of the directors by the relations

$$
\boldsymbol{Q}_{e} \boldsymbol{d}_{i}^{0}=\boldsymbol{d}_{i}, \quad \text { i.e., } \quad \boldsymbol{Q}_{e}=\boldsymbol{d}_{i} \otimes \boldsymbol{d}_{i}^{0} .
$$

Let $\boldsymbol{Q}_{0}$ be the initial microrotation (describing the position of the directors in the reference configuration $\Omega_{\xi}$ )

$$
\boldsymbol{Q}_{0} \boldsymbol{e}_{i}=\boldsymbol{d}_{i}^{0}, \quad \text { i.e., } \quad \boldsymbol{Q}_{0}=\boldsymbol{d}_{i}^{0} \otimes \boldsymbol{e}_{i}
$$

Then, the total microrotation $\overline{\boldsymbol{R}}$ is given by

$$
\overline{\boldsymbol{R}}: \Omega \rightarrow \mathrm{SO}(3), \quad \overline{\boldsymbol{R}}\left(x_{i}\right):=\boldsymbol{Q}_{e}\left(x_{i}\right) \boldsymbol{Q}_{0}\left(x_{i}\right)=\boldsymbol{d}_{i}\left(x_{i}\right) \otimes \boldsymbol{e}_{i} .
$$

The non-symmetric Biot-type stretch tensor (the elastic first Cosserat deformation tensor, see [16], p. 123, eq. (43)) is now

$$
\overline{\boldsymbol{U}}_{e}:=\boldsymbol{Q}_{e}^{T} \boldsymbol{F}=\left(\boldsymbol{d}_{i}^{0} \otimes \boldsymbol{d}_{i}\right)\left(\boldsymbol{\varphi},{ }_{j} \otimes \boldsymbol{g}^{j}\right)=\left(\boldsymbol{\varphi},{ }_{j} \cdot \boldsymbol{d}_{i}\right) \boldsymbol{d}_{i}^{0} \otimes \boldsymbol{g}^{j} .
$$

and the non-symmetric strain tensor for nonlinear micropolar materials is defined by

$$
\overline{\boldsymbol{E}}_{e}:=\overline{\boldsymbol{U}}_{e}-\mathbf{1}_{3}=\left(\boldsymbol{\varphi},{ }_{j} \cdot \boldsymbol{d}_{i}-\boldsymbol{g}_{j} \cdot \boldsymbol{d}_{i}^{0}\right) \boldsymbol{d}_{i}^{0} \otimes \boldsymbol{g}^{j},
$$


where $\mathbf{1}_{3}=\boldsymbol{g}_{i} \otimes \boldsymbol{g}^{i}=\boldsymbol{d}_{i}^{0} \otimes \boldsymbol{d}_{i}^{0}$ is the unit three-dimensional tensor. As a strain measure for curvature (orientation change) one can employ the so-called wryness tensor $\boldsymbol{\Gamma}$ given by :

$$
\boldsymbol{\Gamma}:=\operatorname{axl}\left(\boldsymbol{Q}_{e}^{T} \boldsymbol{Q}_{e, i}\right) \otimes \boldsymbol{g}^{i}=\boldsymbol{Q}_{0}\left[\operatorname{axl}\left(\overline{\boldsymbol{R}}^{T} \overline{\boldsymbol{R}}_{, i}\right)-\operatorname{axl}\left(\boldsymbol{Q}_{0}^{T} \boldsymbol{Q}_{0, i}\right)\right] \otimes \boldsymbol{g}^{i},
$$

where $\operatorname{axl}(\boldsymbol{A})$ denotes the axial vector of any skew-symmetric tensor $\boldsymbol{A}$. For a detailed discussion on various strain measures of nonlinear micropolar continua we refer to the paper 30.

As an alternative to the wryness tensor $\boldsymbol{\Gamma}$ one can make use of the Curl operator to define the so-called dislocation density tensor $\overline{\boldsymbol{D}}_{e}$ by 28 .

$$
\overline{\boldsymbol{D}}_{e}:=\boldsymbol{Q}_{e}^{T} \operatorname{Curl} \boldsymbol{Q}_{e},
$$

which is another curvature measure for micropolar continua. Note that the Curl operator has various definitions in the literature, but we will make its significance clear in the next Subsection 2.1, where we present the Curl operator in curvilinear coordinates. The use of the dislocation density tensor $\overline{\boldsymbol{D}}_{e}$ instead of the wryness tensor in conjuction with micropolar and micromorphic media has several advantages, as it was shown in [20, 27, 24]. The relationship between the wryness tensor $\boldsymbol{\Gamma}$ and the dislocation density tensor $\overline{\boldsymbol{D}}_{e}$ is discussed in the Subsection 2.2 in details.

Using the strain and curvature tensors $\left(\overline{\boldsymbol{E}}_{e}, \overline{\boldsymbol{D}}_{e}\right)$ the elastically stored energy density $W$ for the isotropic nonlinear Cosserat model can be expressed as [26, 22]

$$
\begin{aligned}
W\left(\overline{\boldsymbol{E}}_{e}, \overline{\boldsymbol{D}}_{e}\right) & =W_{\mathrm{mp}}\left(\overline{\boldsymbol{E}}_{e}\right)+W_{\text {curv }}\left(\overline{\boldsymbol{D}}_{e}\right), \quad \text { where } \\
W_{\mathrm{mp}}\left(\overline{\boldsymbol{E}}_{e}\right) & =\mu\left\|\operatorname{dev}_{3} \operatorname{sym} \overline{\boldsymbol{E}}_{e}\right\|^{2}+\mu_{c} \| \text { skew } \overline{\boldsymbol{E}}_{e} \|^{2}+\frac{\kappa}{2}\left(\operatorname{tr} \overline{\boldsymbol{E}}_{e}\right)^{2}, \\
W_{\text {curv }}\left(\overline{\boldsymbol{D}}_{e}\right) & =\mu L_{c}^{p}\left(a_{1}\left\|\operatorname{dev}_{3} \operatorname{sym} \overline{\boldsymbol{D}}_{e}\right\|^{2}+a_{2} \| \text { skew } \overline{\boldsymbol{D}}_{e} \|^{2}+a_{3}\left(\operatorname{tr} \overline{\boldsymbol{D}}_{e}\right)^{2}\right)^{p / 2},
\end{aligned}
$$

where $\mu$ is the shear modulus, $\kappa$ is the bulk modulus of classical isotropic elasticity, and $\mu_{c}$ is called the Cosserat couple modulus, which are assumed to satisfy

$$
\mu>0, \quad \kappa>0, \quad \text { and } \quad \mu_{c}>0 .
$$

The parameter $L_{c}$ introduces an internal length which is characteristic for the material, $a_{i}>0$ are dimensionless constitutive coefficients and $p \geq 2$ is a constant exponent. Here, $\operatorname{dev}_{3} \boldsymbol{X}:=\boldsymbol{X}-\frac{1}{3}(\operatorname{tr} \boldsymbol{X}) \mathbf{1}_{3}$ is the deviatoric part of any second order tensor $\boldsymbol{X}$.

Under these assumptions on the constitutive coefficients the existence of minimizers to the corresponding minimization problem of the total energy functional has been shown, e.g., in $[26,22]$.

\subsection{The Curl operator}

For a vector field $\boldsymbol{v}$, the (coordinate-free) definition of the vector curl $\boldsymbol{v}$ is

$$
(\operatorname{curl} \boldsymbol{v}) \cdot \boldsymbol{c}=\operatorname{div}(\boldsymbol{v} \times \boldsymbol{c}) \quad \text { for all constant vectors } \boldsymbol{c},
$$

where $\cdot$ denotes the scalar product and $\times$ the vector product. The Curl of a tensor field $\boldsymbol{T}$ is the tensor field defined by

$$
(\operatorname{Curl} \boldsymbol{T})^{T} \boldsymbol{c}=\operatorname{curl}\left(\boldsymbol{T}^{T} \boldsymbol{c}\right) \quad \text { for all constant vectors } \boldsymbol{c} .
$$

Remark 2.1. The operator Curl $\boldsymbol{T}$ given by (5) coincides with the Curl operator defined in [32, 25]. However, for other authors the Curl of $\boldsymbol{T}$ is the transpose of Curl $\boldsymbol{T}$ defined by (5), see e.g. [21, 17]. 
Then, from (4) and (5) we obtain the following formulas

$$
\operatorname{curl} \boldsymbol{v}=-\boldsymbol{v}_{, i} \times \boldsymbol{g}^{i}, \quad \operatorname{Curl} \boldsymbol{T}=-\boldsymbol{T}_{, i} \times \boldsymbol{g}^{i} .
$$

Indeed, the definition (4) yields

$$
(\operatorname{curl} \boldsymbol{v}) \cdot \boldsymbol{c}=\operatorname{div}(\boldsymbol{v} \times \boldsymbol{c})=(\boldsymbol{v} \times \boldsymbol{c})_{, i} \cdot \boldsymbol{g}^{i}=(\boldsymbol{v}, i \times \boldsymbol{c}) \cdot \boldsymbol{g}^{i}=\left(\boldsymbol{g}^{i} \times \boldsymbol{v}_{, i}\right) \cdot \boldsymbol{c},
$$

and the equation $(6)_{1}$ holds. Further, from $(5)$ we get

$$
(\operatorname{Curl} \boldsymbol{T})^{T} \boldsymbol{c}=\operatorname{curl}\left(\boldsymbol{T}^{T} \boldsymbol{c}\right)=\boldsymbol{g}^{i} \times\left(\boldsymbol{T}^{T} \boldsymbol{c}\right)_{, i}=\boldsymbol{g}^{i} \times\left(\boldsymbol{T}_{, i}^{T} \boldsymbol{c}\right)=\left(\boldsymbol{g}^{i} \times \boldsymbol{T}_{, i}^{T}\right) \boldsymbol{c}
$$

so it follows $\operatorname{Curl} \boldsymbol{T}=\left(\boldsymbol{g}^{i} \times \boldsymbol{T}_{, i}^{T}\right)^{T}=-\boldsymbol{T}_{, i} \times \boldsymbol{g}^{i}$ and the relations 6 are proved.

In order to write the components of $\operatorname{curl} \boldsymbol{v}$ and $\operatorname{Curl} \boldsymbol{T}$ in curvilinear coordinates, we introduce the following notations

$$
g_{i j}=\boldsymbol{g}_{i} \otimes \boldsymbol{g}_{j}, \quad g=\operatorname{det}\left(g_{i j}\right)_{3 \times 3}>0 .
$$

The alternating (Ricci) third-order tensor is

$$
\begin{array}{r}
\boldsymbol{\epsilon}=-\mathbf{1}_{3} \times \mathbf{1}_{3}=\epsilon_{i j k} \boldsymbol{g}^{i} \otimes \boldsymbol{g}^{j} \otimes \boldsymbol{g}^{k}=\epsilon^{i j k} \boldsymbol{g}_{i} \otimes \boldsymbol{g}_{j} \otimes \boldsymbol{g}_{k}, \quad \text { where } \\
\epsilon_{i j k}=\sqrt{g} e_{i j k}, \quad \epsilon^{i j k}=\frac{1}{\sqrt{g}} e_{i j k}, \quad e_{i j k}=\left\{\begin{aligned}
1, & (i, j, k) \text { is even permutation } \\
-1, & (i, j, k) \text { is odd permutation } \\
0, & (i, j, k) \text { is no permutation }
\end{aligned}\right.
\end{array}
$$

The covariant, contravariant, and mixed components of any vector field $\boldsymbol{v}$ and any tensor field $\boldsymbol{T}$ are introduced by

$$
\boldsymbol{v}=v_{k} \boldsymbol{g}^{k}=v^{k} \boldsymbol{g}_{k}, \quad \boldsymbol{T}=T_{j k} \boldsymbol{g}^{j} \otimes \boldsymbol{g}^{k}=T^{j k} \boldsymbol{g}_{j} \otimes \boldsymbol{g}_{k}=T_{\cdot k}^{j} \boldsymbol{g}_{j} \otimes \boldsymbol{g}^{k} .
$$

For the partial derivatives with respect to $x_{i}$ we have the well-known expressions

$$
\boldsymbol{v}_{, i}=v_{k \mid i} \boldsymbol{g}^{k}, \quad \boldsymbol{T}_{, i}=T_{j k \mid i} \boldsymbol{g}^{j} \otimes \boldsymbol{g}^{k}=T_{. k \mid i}^{j} \boldsymbol{g}_{j} \otimes \boldsymbol{g}^{k},
$$

where a subscript bar preceding the index $i$ denotes covariant derivative w.r.t. $x_{i}$.

Using the relations (7) in (6) we can write the components of curl $\boldsymbol{v}$ and $\operatorname{Curl} \boldsymbol{T}$ as follows

$$
\operatorname{curl} \boldsymbol{v}=\epsilon^{i j k} v_{j \mid i} \boldsymbol{g}_{k}, \quad \operatorname{Curl} \boldsymbol{T}=\epsilon^{i j k} T_{s j \mid i} \boldsymbol{g}^{s} \otimes \boldsymbol{g}_{k}=\epsilon^{i j k} T_{\cdot j \mid i}^{s} \boldsymbol{g}_{s} \otimes \boldsymbol{g}_{k} .
$$

Indeed, from $(6)_{1}$ and $(7)_{1}$ we find

$$
\operatorname{curl} \boldsymbol{v}=-\left(v_{k \mid i} \boldsymbol{g}^{k}\right) \times \boldsymbol{g}^{i}=-v_{k \mid i}\left(\boldsymbol{g}^{k} \times \boldsymbol{g}^{i}\right)=-v_{k \mid i}\left(\epsilon^{k i j} \boldsymbol{g}_{j}\right)=\epsilon^{i j k} v_{j \mid i} \boldsymbol{g}_{k} .
$$

Analogously, from $(6)_{2}$ and $(7)_{2}$ we get

$$
\operatorname{Curl} \boldsymbol{T}=-\left(T_{s k \mid i} \boldsymbol{g}^{s} \otimes \boldsymbol{g}^{k}\right) \times \boldsymbol{g}^{i}=-T_{s k \mid i} \boldsymbol{g}^{s} \otimes\left(\boldsymbol{g}^{k} \times \boldsymbol{g}^{i}\right)=\epsilon^{i j k} T_{s j \mid i} \boldsymbol{g}^{s} \otimes \boldsymbol{g}_{k} .
$$

Thus the equations 8 are proved.

Remark 2.2. In the special case of Cartesian coordinates, the relations (6) and (8) admit the simple form

$$
\operatorname{curl} \boldsymbol{v}=-\boldsymbol{v}_{, i} \times \boldsymbol{e}_{i}=e_{i j k} v_{j, i} \boldsymbol{e}_{k}, \quad \operatorname{Curl} \boldsymbol{T}=-\boldsymbol{T}_{, i} \times \boldsymbol{e}_{i}=e_{i j k} T_{s j, i} \boldsymbol{e}_{s} \otimes \boldsymbol{e}_{k},
$$

where $\boldsymbol{v}=v_{i} \boldsymbol{e}_{i}$ and $\boldsymbol{T}=T_{i j} \boldsymbol{e}_{i} \otimes \boldsymbol{e}_{j}$ are the corresponding coordinates. Moreover, in this case one can write

$$
\operatorname{Curl} \boldsymbol{T}=\boldsymbol{e}_{i} \otimes \operatorname{curl}\left(\boldsymbol{T}_{i}\right) \quad \text { for } \quad \boldsymbol{T}=\boldsymbol{e}_{i} \otimes \boldsymbol{T}_{i},
$$

where $\boldsymbol{T}_{i}=T_{i j} \boldsymbol{e}_{j}$ are the three rows of the $3 \times 3$ matrix $\left(T_{i j}\right)_{3 \times 3}$. The relation 9 shows that Curl is defined row-wise [28]: the rows of the $3 \times 3$ matrix $\operatorname{Curl} \boldsymbol{T}$ are respectively the three vectors $\operatorname{curl}\left(\boldsymbol{T}_{i}\right), \quad i=1,2,3$. 
Remark 2.3. In order to write the corresponding formula in curvilinear coordinates which is analogous to (9), we introduce the vectors $\boldsymbol{T}_{i}:=T_{i j} \boldsymbol{g}^{j}$ and $\boldsymbol{T}^{i}:=T^{i j} \boldsymbol{g}_{j}=T_{. j}^{i} \boldsymbol{g}^{j}$ such that it holds

$$
\boldsymbol{T}=\boldsymbol{g}^{i} \otimes \boldsymbol{T}_{i} \quad \text { and } \quad \boldsymbol{T}=\boldsymbol{g}_{i} \otimes \boldsymbol{T}^{i} .
$$

If we differentiate $10 p_{1}$ with respect to $x_{j}$ we get

$$
\boldsymbol{T}_{, j}=\boldsymbol{g}_{\cdot, j}^{r} \otimes \boldsymbol{T}_{r}+\boldsymbol{g}^{i} \otimes \boldsymbol{T}_{i, j}=-\Gamma_{j i}^{r} \boldsymbol{g}^{i} \otimes \boldsymbol{T}_{r}+\boldsymbol{g}^{i} \otimes \boldsymbol{T}_{i, j}=\boldsymbol{g}^{i} \otimes\left(\boldsymbol{T}_{i, j}-\Gamma_{j i}^{r} \boldsymbol{T}_{r}\right),
$$

where $\Gamma_{i j}^{r}$ are the Christoffel symbols of the second kind. Hence, it follows

$$
\boldsymbol{T}_{, j}=\boldsymbol{g}^{i} \otimes \boldsymbol{T}_{i \mid j} \quad \text { with } \quad \boldsymbol{T}_{i \mid j}:=\boldsymbol{T}_{i, j}-\Gamma_{j i}^{r} \boldsymbol{T}_{r}=T_{i k \mid j} \boldsymbol{g}^{k} .
$$

Taking the vector product of 11,1 with $\boldsymbol{g}^{j}$ we obtain

$$
\begin{gathered}
\operatorname{Curl} \boldsymbol{T}=-\boldsymbol{T}_{, j} \times \boldsymbol{g}^{j}=-\left(\boldsymbol{g}^{i} \otimes \boldsymbol{T}_{i \mid j}\right) \times \boldsymbol{g}^{j}, \quad \text { i.e. } \\
\operatorname{Curl} \boldsymbol{T}=\boldsymbol{g}^{i} \otimes \operatorname{curl}_{\mathrm{cov}}\left(\boldsymbol{T}_{i}\right) \quad \text { where } \quad \operatorname{curl}_{\mathrm{cov}}\left(\boldsymbol{T}_{i}\right):=-\boldsymbol{T}_{i \mid j} \times \boldsymbol{g}^{j} .
\end{gathered}
$$

The relation 12 is the analogue of $(9)$ for curvilinear coordinates. Similarly, by differentiating 10$]_{2}$ with respect to $x_{j}$ one can obtain the relation

$$
\begin{gathered}
\operatorname{Curl} \boldsymbol{T}=\boldsymbol{g}_{i} \otimes \operatorname{curl}_{\mathrm{cov}}\left(\boldsymbol{T}^{i}\right) \quad \text { where we denote } \\
\operatorname{curl}_{\mathrm{cov}}\left(\boldsymbol{T}^{i}\right):=-\boldsymbol{T}_{\cdot{ }_{\mid j}}^{i} \times \boldsymbol{g}^{j} \quad \text { and } \quad \boldsymbol{T}_{\cdot{ }_{\mid j}}^{i}:=\boldsymbol{T}_{\cdot, j}^{i}+\Gamma_{r j}^{i} \boldsymbol{T}^{r}=T_{\cdot k \mid j}^{i} \boldsymbol{g}^{k} .
\end{gathered}
$$

\subsection{Relation between the wryness tensor and the dislocation density tensor}

Let $\boldsymbol{A}=A_{i j} \boldsymbol{g}^{i} \otimes \boldsymbol{g}^{j}$ be an arbitrary skew-symmetric tensor and $\operatorname{axl}(\boldsymbol{A})=a_{k} \boldsymbol{g}^{k}$ its axial vector. Then, the following relations hold

$$
\begin{array}{ll}
\boldsymbol{A} & =\operatorname{axl}(\boldsymbol{A}) \times \mathbf{1}_{3}=\mathbf{1}_{3} \times \operatorname{axl}(\boldsymbol{A}), \\
\operatorname{axl}(\boldsymbol{A}) & =-\frac{1}{2} \boldsymbol{\epsilon}: \boldsymbol{A}=-\frac{1}{2} \epsilon^{i j k} A_{i j} \boldsymbol{g}_{k}, \\
\boldsymbol{A} & =-\boldsymbol{\epsilon} \operatorname{axl}(\boldsymbol{A})=-\epsilon^{i j k} a_{k} \boldsymbol{g}_{i} \otimes \boldsymbol{g}_{j},
\end{array}
$$

where the double dot product " : " of two tensors $\boldsymbol{B}=B^{i j k} \boldsymbol{g}_{i} \otimes \boldsymbol{g}_{j} \otimes \boldsymbol{g}_{k}$ and $\boldsymbol{T}=T_{i j} \boldsymbol{g}^{i} \otimes \boldsymbol{g}^{j}$ is defined as $\boldsymbol{B}: \boldsymbol{T}=B^{i j k} T_{j k} \boldsymbol{g}_{i}$.

Using these relations we can derive the close relationship between the wryness tensor and the dislocation density tensor: it holds

$$
\begin{gathered}
\overline{\boldsymbol{D}}_{e}=-\boldsymbol{\Gamma}^{T}+(\operatorname{tr} \boldsymbol{\Gamma}) \mathbf{1}_{3}, \quad \text { or equivalently, } \\
\boldsymbol{\Gamma}=-\overline{\boldsymbol{D}}_{e}^{T}+\frac{1}{2}\left(\operatorname{tr} \overline{\boldsymbol{D}}_{e}\right) \mathbf{1}_{3} .
\end{gathered}
$$

Indeed, in view of the equation $(14)_{3}$ and the definition (1) we have

$$
\begin{aligned}
\boldsymbol{Q}_{e}^{T} \boldsymbol{Q}_{e, k} \otimes \boldsymbol{g}^{k} & =-\boldsymbol{\epsilon} \operatorname{axl}\left(Q_{e}^{T} \boldsymbol{Q}_{e, k}\right) \otimes \boldsymbol{g}^{k}=-\boldsymbol{\epsilon} \boldsymbol{\Gamma} \\
& =-\left(\epsilon_{i j r} \boldsymbol{g}^{i} \otimes \boldsymbol{g}^{j} \otimes \boldsymbol{g}^{r}\right)\left(\Gamma_{\cdot k}^{s} \boldsymbol{g}_{s} \otimes \boldsymbol{g}^{k}\right)=-\epsilon_{i j s} \Gamma_{. k}^{s} \boldsymbol{g}^{i} \otimes \boldsymbol{g}^{j} \otimes \boldsymbol{g}^{k} .
\end{aligned}
$$

Hence, we deduce

$$
\boldsymbol{Q}_{e}^{T} \boldsymbol{Q}_{e, k}=-\epsilon_{i j s} \Gamma_{. k}^{s} \boldsymbol{g}^{i} \otimes \boldsymbol{g}^{j} .
$$

In view of $(6)_{2}$, the definition $(2)$ can be written in the form

$$
\overline{\boldsymbol{D}}_{e}=\boldsymbol{Q}_{e}^{T}\left(-\boldsymbol{Q}_{e, k} \times \boldsymbol{g}^{k}\right)=-\left(\boldsymbol{Q}_{e}^{T} \boldsymbol{Q}_{e, k}\right) \times \boldsymbol{g}^{k} .
$$


Inserting (17) in (18), we obtain

$$
\begin{aligned}
\overline{\boldsymbol{D}}_{e} & =\epsilon_{i j s} \Gamma_{\cdot k}^{s}\left(\boldsymbol{g}^{i} \otimes \boldsymbol{g}^{j}\right) \times \boldsymbol{g}^{k}=\epsilon_{i j s} \Gamma_{\cdot k}^{s} \boldsymbol{g}^{i} \otimes\left(\epsilon^{j k r} \boldsymbol{g}_{r}\right)=\left(\epsilon_{j s i} \epsilon^{j k r}\right) \Gamma_{\cdot k}^{s} \boldsymbol{g}^{i} \otimes \boldsymbol{g}_{r} \\
& =\left(\delta_{s}^{k} \delta_{i}^{r}-\delta_{s}^{r} \delta_{i}^{k}\right) \Gamma_{\cdot k}^{s} \boldsymbol{g}^{i} \otimes \boldsymbol{g}_{r}=\Gamma_{\cdot s}^{s} \boldsymbol{g}^{i} \otimes \boldsymbol{g}_{i}-\Gamma_{\cdot i}^{s} \boldsymbol{g}^{i} \otimes \boldsymbol{g}_{s}=(\operatorname{tr} \boldsymbol{\Gamma}) \mathbf{1}_{3}-\boldsymbol{\Gamma}^{T}
\end{aligned}
$$

Thus, the relation 15 is proved. If we apply the trace operator and the transpose in 15 we obtain also the relation (16). For infinitesimal strains this formula is well-known under the name Nye's formula, and $(-\boldsymbol{\Gamma})$ is also called Nye's curvature tensor 29 . This relation has been first established in [28].

Let us find the components of the wryness tensor and the dislocation density tensor in curvilinear coordinates. To this aim, we write first the sqew-symmetric tensor

$$
\begin{aligned}
\boldsymbol{Q}_{e}^{T} \boldsymbol{Q}_{e, i} & =\left(\boldsymbol{d}_{j}^{0} \otimes \boldsymbol{d}_{j}\right)\left(\boldsymbol{d}_{k, i} \otimes \boldsymbol{d}_{k}^{0}+\boldsymbol{d}_{k} \otimes \boldsymbol{d}_{k, i}^{0}\right)=\left(\boldsymbol{d}_{j} \cdot \boldsymbol{d}_{k, i}\right) \boldsymbol{d}_{j}^{0} \otimes \boldsymbol{d}_{k}^{0}+\boldsymbol{d}_{j}^{0} \otimes \boldsymbol{d}_{j, i}^{0} \\
& =\left(\boldsymbol{d}_{j} \cdot \boldsymbol{d}_{k, i}-\boldsymbol{d}_{j}^{0} \cdot \boldsymbol{d}_{k, i}^{0}\right) \boldsymbol{d}_{j}^{0} \otimes \boldsymbol{d}_{k}^{0}
\end{aligned}
$$

Then, we obtain for the axial vector the equation

$$
\operatorname{axl}\left(\boldsymbol{Q}_{e}^{T} \boldsymbol{Q}_{e, i}\right)=-\frac{1}{2} e_{j k s}\left(\boldsymbol{d}_{j} \cdot \boldsymbol{d}_{k, i}-\boldsymbol{d}_{j}^{0} \cdot \boldsymbol{d}_{k, i}^{0}\right) \boldsymbol{d}_{s}^{0} .
$$

Indeed, according to $14{ }_{2}$ and 19 we can write

$$
\begin{aligned}
\operatorname{axl}\left(\boldsymbol{Q}_{e}^{T} \boldsymbol{Q}_{e, i}\right) & =-\frac{1}{2} \boldsymbol{\epsilon}:\left(\boldsymbol{Q}_{e}^{T} \boldsymbol{Q}_{e, i}\right) \\
& =-\frac{1}{2}\left(e_{s j k} \boldsymbol{d}_{s}^{0} \otimes \boldsymbol{d}_{j}^{0} \otimes \boldsymbol{d}_{k}^{0}\right):\left[\left(\boldsymbol{d}_{l} \cdot \boldsymbol{d}_{r, i}-\boldsymbol{d}_{l}^{0} \cdot \boldsymbol{d}_{r, i}^{0}\right) \boldsymbol{d}_{l}^{0} \otimes \boldsymbol{d}_{r}^{0}\right] \\
& =-\frac{1}{2} e_{j k s}\left(\boldsymbol{d}_{j} \cdot \boldsymbol{d}_{k, i}-\boldsymbol{d}_{j}^{0} \cdot \boldsymbol{d}_{k, i}^{0}\right) \boldsymbol{d}_{s}^{0}
\end{aligned}
$$

and the relation 20 is proved. Using 200 in the definition (1) we find the following formula for the wryness tensor

$$
\boldsymbol{\Gamma}=\frac{1}{2} e_{j k s}\left(\boldsymbol{d}_{j, i} \cdot \boldsymbol{d}_{k}-\boldsymbol{d}_{j, i}^{0} \cdot \boldsymbol{d}_{k}^{0}\right) \boldsymbol{d}_{s}^{0} \otimes \boldsymbol{g}^{i}
$$

To obtain an expression for the components of $\overline{\boldsymbol{D}}_{e}$ we insert 19 in 18 and we get

$$
\begin{aligned}
\overline{\boldsymbol{D}}_{e} & =-\left(\boldsymbol{d}_{j} \cdot \boldsymbol{d}_{k, i}-\boldsymbol{d}_{j}^{0} \cdot \boldsymbol{d}_{k, i}^{0}\right)\left(\boldsymbol{d}_{j}^{0} \otimes \boldsymbol{d}_{k}^{0}\right) \times \boldsymbol{g}^{i} \\
& =\left(\boldsymbol{d}_{j, i} \cdot \boldsymbol{d}_{k}-\boldsymbol{d}_{j, i}^{0} \cdot \boldsymbol{d}_{k}^{0}\right) \boldsymbol{d}_{j}^{0} \otimes\left(\boldsymbol{d}_{k}^{0} \times \boldsymbol{g}^{i}\right) .
\end{aligned}
$$

We rewrite the last vector product as

$$
\boldsymbol{d}_{k}^{0} \times \boldsymbol{g}^{i}=\boldsymbol{d}_{k}^{0} \times\left[\left(\boldsymbol{g}^{i} \cdot \boldsymbol{d}_{r}^{0}\right) \boldsymbol{d}_{r}^{0}\right]=\left(\boldsymbol{g}^{i} \cdot \boldsymbol{d}_{r}^{0}\right) \boldsymbol{d}_{k}^{0} \times \boldsymbol{d}_{r}^{0}=e_{k r s}\left(\boldsymbol{g}^{i} \cdot \boldsymbol{d}_{r}^{0}\right) \boldsymbol{d}_{s}^{0}
$$

and we insert it in 22 to find the following expression for the dislocation density tensor

$$
\bar{D}_{e}=e_{k r s}\left(\boldsymbol{d}_{j, i} \cdot \boldsymbol{d}_{k}-\boldsymbol{d}_{j, i}^{0} \cdot \boldsymbol{d}_{k}^{0}\right)\left(\boldsymbol{g}^{i} \cdot \boldsymbol{d}_{r}^{0}\right) \boldsymbol{d}_{j}^{0} \otimes \boldsymbol{d}_{s}^{0} .
$$

Remark 2.4. In the special case of Cartesian coordinates one can identify $\boldsymbol{d}_{i}^{0}=\boldsymbol{e}_{i}, \boldsymbol{g}^{i}=$ $\boldsymbol{g}_{i}=\boldsymbol{e}_{i}$, and the relations (21) and 22 simplify to the forms

$$
\begin{aligned}
\boldsymbol{\Gamma} & =\frac{1}{2} e_{i k s}\left(\boldsymbol{d}_{k, j} \cdot \boldsymbol{d}_{s}\right) \boldsymbol{e}_{i} \otimes \boldsymbol{e}_{j}, \\
\overline{\boldsymbol{D}}_{e} & =e_{i j k}\left(\boldsymbol{d}_{j, i} \cdot \boldsymbol{d}_{s}\right) \boldsymbol{e}_{s} \otimes \boldsymbol{e}_{k} .
\end{aligned}
$$


Remark 2.5. One can find various definitions of the wryness tensor in the literature, see e.g. 33, where $\boldsymbol{\Gamma}$ is called the curvature strain tensor. Thus, one can alternatively define the wryness tensor by

$$
\boldsymbol{\Gamma}=\boldsymbol{Q}_{e}^{T} \boldsymbol{\omega}
$$

where $\boldsymbol{\omega}$ is the second order tensor given by

$$
\boldsymbol{\omega}=\boldsymbol{\omega}_{i} \otimes \boldsymbol{g}^{i} \quad \text { with } \quad \boldsymbol{Q}_{e, i}=\boldsymbol{\omega}_{i} \times \boldsymbol{Q}_{e} .
$$

If we compare the definition (1) with 24, 25, we see that indeed $\boldsymbol{Q}_{e}^{T} \boldsymbol{\omega}_{i}=\operatorname{axl}\left(\boldsymbol{Q}_{e}^{T} \boldsymbol{Q}_{e, i}\right)$, i.e.

$$
\boldsymbol{\omega}_{i}=\boldsymbol{Q}_{e} \operatorname{axl}\left(\boldsymbol{Q}_{e}^{T} \boldsymbol{Q}_{e, i}\right)=\operatorname{axl}\left(\boldsymbol{Q}_{e, i} \boldsymbol{Q}_{e}^{T}\right) .
$$

By a straightforward but lengthy calculation one can prove that the vectors $\boldsymbol{\omega}_{i}$ are expressed in terms of the directors by

$$
\boldsymbol{\omega}_{i}=\frac{1}{2}\left[\boldsymbol{d}_{j} \times \boldsymbol{d}_{j, i}-\boldsymbol{Q}_{e}\left(\boldsymbol{d}_{j}^{0} \times \boldsymbol{d}_{j, i}^{0}\right)\right] .
$$

Inserting (27) in 25, and 24 we obtain the expression of the wryness tensor written with the help of the directors $\boldsymbol{d}_{i}$

$$
\boldsymbol{\Gamma}=\frac{1}{2}\left[\boldsymbol{Q}_{e}^{T}\left(\boldsymbol{d}_{j} \times \boldsymbol{d}_{j, i}\right)-\boldsymbol{d}_{j}^{0} \times \boldsymbol{d}_{j, i}^{0}\right] \otimes \boldsymbol{g}^{i}
$$

\section{The Curl operator on surfaces}

Let $\mathcal{S}$ be a smooth surface embedded in the Euclidean space $\mathbb{R}^{3}$ and let $\boldsymbol{y}_{0}\left(x_{1}, x_{2}\right), \boldsymbol{y}_{0}$ : $\omega \rightarrow \mathbb{R}^{3}$, be a parametrization of this surface. We denote the covariant base vectors in the tangent plane by $\boldsymbol{a}_{1}, \boldsymbol{a}_{2}$ and the contravariant base vectors by $\boldsymbol{a}^{1}, \boldsymbol{a}^{2}$ :

$$
\boldsymbol{a}_{\alpha}=\frac{\partial \boldsymbol{y}_{0}}{\partial x_{\alpha}}=\boldsymbol{y}_{0, \alpha}, \quad \boldsymbol{a}_{\alpha} \cdot \boldsymbol{a}^{\beta}=\delta_{\alpha}^{\beta}
$$

and let

$$
\boldsymbol{a}_{3}=\boldsymbol{a}^{3}=\boldsymbol{n}_{0}=\frac{\boldsymbol{a}_{1} \times \boldsymbol{a}_{2}}{\left|\boldsymbol{a}_{1} \times \boldsymbol{a}_{2}\right|}
$$

where $\boldsymbol{n}_{0}$ is the unit normal to the surface. Further, we designate by

$$
a_{\alpha \beta}=\boldsymbol{a}_{\alpha} \cdot \boldsymbol{a}_{\beta}, \quad a^{\alpha \beta}=\boldsymbol{a}^{\alpha} \cdot \boldsymbol{a}^{\beta}, \quad a=\sqrt{\operatorname{det}\left(a_{\alpha \beta}\right)_{2 \times 2}}=\left|\boldsymbol{a}_{1} \times \boldsymbol{a}_{2}\right|>0
$$

and we have

$$
\boldsymbol{a}^{\alpha} \times \boldsymbol{a}^{\beta}=\epsilon^{\alpha \beta} \boldsymbol{a}_{3}, \quad \boldsymbol{a}^{3} \times \boldsymbol{a}^{\alpha}=\epsilon^{\alpha \beta} \boldsymbol{a}_{\beta}, \quad \boldsymbol{a}_{\alpha} \times \boldsymbol{a}_{\beta}=\epsilon_{\alpha \beta} \boldsymbol{a}^{3}, \quad \boldsymbol{a}_{3} \times \boldsymbol{a}_{\alpha}=\epsilon_{\alpha \beta} \boldsymbol{a}^{\beta},
$$

where $\epsilon^{\alpha \beta}=\frac{1}{a} e_{\alpha \beta}, \epsilon_{\alpha \beta}=a e_{\alpha \beta}$ and $e_{\alpha \beta}$ is the two-dimensional alternator given by $e_{12}=$ $-e_{21}=1, e_{11} \stackrel{a}{=} e_{22}=0$.

Then, $\boldsymbol{a}=a_{\alpha \beta} \boldsymbol{a}^{\alpha} \otimes \boldsymbol{a}^{\beta}=a^{\alpha \beta} \boldsymbol{a}_{\alpha} \otimes \boldsymbol{a}_{\beta}=\boldsymbol{a}_{\alpha} \otimes \boldsymbol{a}^{\alpha}$ represents the first fundamental tensor of the surface $\mathcal{S}$, while the second fundamental tensor $\boldsymbol{b}$ is defined by

$$
\begin{aligned}
& \boldsymbol{b}=-\operatorname{Grad}_{s} \boldsymbol{n}_{0}=-\boldsymbol{n}_{0, \alpha} \otimes \boldsymbol{a}^{\alpha}=b_{\alpha \beta} \boldsymbol{a}^{\alpha} \otimes \boldsymbol{a}^{\beta}=b_{\beta}^{\alpha} \boldsymbol{a}_{\alpha} \otimes \boldsymbol{a}^{\beta}, \quad \text { with } \\
& b_{\alpha \beta}=-\boldsymbol{n}_{0, \beta} \cdot \boldsymbol{a}_{\alpha}=b_{\beta \alpha}, \quad b_{\beta}^{\alpha}=-\boldsymbol{n}_{0, \beta} \cdot \boldsymbol{a}^{\alpha} .
\end{aligned}
$$


The surface gradient $\operatorname{Grad}_{s}$ and surface divergence Div $s$ operators are defined for a vector field $v$ by

$$
\operatorname{Grad}_{s} \boldsymbol{v}=\frac{\partial \boldsymbol{v}}{\partial x_{\alpha}} \otimes \boldsymbol{a}^{\alpha}=\boldsymbol{v}_{, \alpha} \otimes \boldsymbol{a}^{\alpha}, \quad \operatorname{Div}_{s} \boldsymbol{v}=\operatorname{tr}\left[\operatorname{Grad}_{s} \boldsymbol{v}\right]=\boldsymbol{v}_{, \alpha} \cdot \boldsymbol{a}^{\alpha}
$$

We also introduce the so-called alternator tensor $\boldsymbol{c}$ of the surface 35

$$
\boldsymbol{c}=-\boldsymbol{n}_{0} \times \boldsymbol{a}=-\boldsymbol{a} \times \boldsymbol{n}_{0}=\epsilon^{\alpha \beta} \boldsymbol{a}_{\alpha} \otimes \boldsymbol{a}_{\beta}=\epsilon_{\alpha \beta} \boldsymbol{a}^{\alpha} \otimes \boldsymbol{a}^{\beta} .
$$

The tensors $\boldsymbol{a}$ and $\boldsymbol{b}$ are symmetric, while $\boldsymbol{c}$ is skew-symmetric and satisfies $\boldsymbol{c} \boldsymbol{c}=-\boldsymbol{a}$. Note that the tensors $\boldsymbol{a}, \boldsymbol{b}$, and $\boldsymbol{c}$ defined above are planar, i.e. they are tensors in the tangent plane of the surface. Moreover, $\boldsymbol{a}$ is the identity tensor in the tangent plane.

We define the surface Curl operator $\mathrm{curl}_{s}$ for vector fields $\boldsymbol{v}$ and, respectively, $\mathrm{Curl}_{s}$ for tensor fields $\boldsymbol{T}$ by

$$
\begin{aligned}
& \left(\operatorname{curl}_{s} \boldsymbol{v}\right) \cdot \boldsymbol{k}=\operatorname{Div}_{s}(\boldsymbol{v} \times \boldsymbol{k}) \quad \text { for all constant vectors } \boldsymbol{k}, \\
& \left(\operatorname{Curl}_{s} \boldsymbol{T}\right)^{T} \boldsymbol{k}=\operatorname{curl}_{s}\left(\boldsymbol{T}^{T} \boldsymbol{k}\right) \quad \text { for all constant vectors } \boldsymbol{k} .
\end{aligned}
$$

Thus, $\operatorname{curl}_{s} \boldsymbol{v}$ is a vector field, while $\operatorname{Curl}_{s} \boldsymbol{T}$ is a tensor field.

Remark 3.1. These definitions are analogous to the corresponding definitions (4), (5) in the three-dimensional case. Notice that the curl operator on surfaces has a different significance for other authors, see e.g. [9].

From the definitions 32 and 33 it follows

$$
\operatorname{curl}_{s} \boldsymbol{v}=-\boldsymbol{v},_{\alpha} \times \boldsymbol{a}^{\alpha}, \quad \mathrm{Curl}_{s} \boldsymbol{T}=-\boldsymbol{T},_{\alpha} \times \boldsymbol{a}^{\alpha} .
$$

Indeed, in view of 30 and 32 we have

$$
\begin{aligned}
\left(\operatorname{curl}_{s} \boldsymbol{v}\right) \cdot \boldsymbol{k} & =\operatorname{Div}_{s}(\boldsymbol{v} \times \boldsymbol{k})=(\boldsymbol{v} \times \boldsymbol{k})_{, \alpha} \cdot \boldsymbol{a}^{\alpha}=\left(\boldsymbol{v},_{\alpha} \times \boldsymbol{k}\right) \cdot \boldsymbol{a}^{\alpha} \\
& =\left(\boldsymbol{a}^{\alpha} \times \boldsymbol{v}, \alpha\right) \cdot \boldsymbol{k}=\left(-\boldsymbol{v}, \alpha \times \boldsymbol{a}^{\alpha}\right) \cdot \boldsymbol{k} \text { for all constant vectors } \boldsymbol{k}
\end{aligned}
$$

and also

$$
\left(\operatorname{Curl}_{s} \boldsymbol{T}\right)^{T} \boldsymbol{k}=\operatorname{curl}_{s}\left(\boldsymbol{T}^{T} \boldsymbol{k}\right)=\boldsymbol{a}^{\alpha} \times\left(\boldsymbol{T}^{T} \boldsymbol{k}\right)_{, \alpha}=\boldsymbol{a}^{\alpha} \times\left(\boldsymbol{T}_{, \alpha}^{T} \boldsymbol{k}\right)=\left(\boldsymbol{a}^{\alpha} \times \boldsymbol{T}_{, \alpha}^{T}\right) \boldsymbol{k},
$$

which implies $\operatorname{Curl}_{s} \boldsymbol{T}=\left(\boldsymbol{a}^{\alpha} \times \boldsymbol{T}_{, \alpha}^{T}\right)^{T}=-\boldsymbol{T}_{, \alpha} \times \boldsymbol{a}^{\alpha}$, so the relations 34 hold true.

To write the components of $\operatorname{curl}_{s} \boldsymbol{v}$ and $\operatorname{Curl}_{s} \boldsymbol{T}$ we employ the covariant derivatives on the surface. Let $\boldsymbol{v}=v_{i} \boldsymbol{a}^{i}$ be a vector field on $\mathcal{S}$. Then, we have

$$
\begin{aligned}
& \boldsymbol{a}_{\cdot, \beta}^{\alpha}=-\Gamma_{\beta \gamma}^{\alpha} \boldsymbol{a}^{\gamma}+b_{\beta}^{\alpha} \boldsymbol{a}^{3}, \quad \boldsymbol{a}_{3, \beta}=-b_{\beta}^{\alpha} \boldsymbol{a}_{\alpha}=-b_{\alpha \beta} \boldsymbol{a}^{\alpha}, \\
& \boldsymbol{v}_{, \alpha}=\left(v_{\beta \mid \alpha}-b_{\alpha \beta} v_{3}\right) \boldsymbol{a}^{\beta}+\left(v_{3, \alpha}+b_{\alpha}^{\beta} v_{\beta}\right) \boldsymbol{a}^{3},
\end{aligned}
$$

where $v_{\beta \mid \alpha}=v_{\beta, \alpha}-\Gamma_{\alpha \beta}^{\gamma} v_{\gamma}$ is the covariant derivative with respect to $x_{\alpha}$. Inserting this relation in 34$)_{1}$ and using 29$)_{1,2}$ we obtain

$$
\operatorname{curl}_{s} \boldsymbol{v}=\epsilon^{\alpha \beta}\left[\left(v_{3, \beta}+b_{\beta}^{\gamma} v_{\gamma}\right) \boldsymbol{a}_{\alpha}+v_{\beta \mid \alpha} \boldsymbol{a}_{3}\right] .
$$

For a tensor field $\boldsymbol{T}=T_{i j} \boldsymbol{a}^{i} \otimes \boldsymbol{a}^{j}=T^{i j} \boldsymbol{a}_{i} \otimes \boldsymbol{a}_{j}=T_{. j}^{i} \boldsymbol{a}_{i} \otimes \boldsymbol{a}^{j}$ on the surface, the derivative $\boldsymbol{T}_{, \gamma}$ can be expressed as

$$
\begin{aligned}
\boldsymbol{T}_{, \gamma}= & \left(T_{\alpha \beta \mid \gamma}-b_{\alpha \gamma} T_{3 \beta}-b_{\beta \gamma} T_{\alpha 3}\right) \boldsymbol{a}^{\alpha} \otimes \boldsymbol{a}^{\beta}+\left(T_{\alpha 3 \mid \gamma}+b_{\gamma}^{\beta} T_{\alpha \beta}-b_{\alpha \gamma} T_{33}\right) \boldsymbol{a}^{\alpha} \otimes \boldsymbol{a}^{3} \\
& +\left(T_{3 \alpha \mid \gamma}+b_{\gamma}^{\beta} T_{\beta \alpha}-b_{\alpha \gamma} T_{33}\right) \boldsymbol{a}^{3} \otimes \boldsymbol{a}^{\alpha}+\left(T_{33, \gamma}+b_{\gamma}^{\alpha} T_{\alpha 3}+b_{\gamma}^{\alpha} T_{3 \alpha}\right) \boldsymbol{a}^{3} \otimes \boldsymbol{a}^{3},
\end{aligned}
$$


where the covariant derivatives are

$$
\begin{aligned}
& T_{\alpha \beta \mid \gamma}=T_{\alpha \beta, \gamma}-\Gamma_{\beta \gamma}^{\delta} T_{\alpha \delta}-\Gamma_{\alpha \gamma}^{\delta} T_{\delta \beta}, \\
& T_{\alpha 3 \mid \gamma}=T_{\alpha 3, \gamma}-\Gamma_{\alpha \gamma}^{\beta} T_{\beta 3}, \quad T_{3 \alpha \mid \gamma}=T_{3 \alpha, \gamma}-\Gamma_{\alpha \gamma}^{\beta} T_{3 \beta} .
\end{aligned}
$$

Using (37) in 34$]_{2}$ we obtain with the help of 29$)_{1,2}$

$$
\begin{aligned}
\operatorname{Curl}_{s} \boldsymbol{T} & =\epsilon^{\beta \gamma}\left(T_{\alpha 3 \mid \gamma}+b_{\gamma}^{\sigma} T_{\alpha \sigma}-b_{\alpha \gamma} T_{33}\right) \boldsymbol{a}^{\alpha} \otimes \boldsymbol{a}_{\beta}+\epsilon^{\gamma \beta}\left(T_{\alpha \beta \mid \gamma}-b_{\alpha \gamma} T_{3 \beta}\right) \boldsymbol{a}^{\alpha} \otimes \boldsymbol{a}_{3} \\
& +\epsilon^{\beta \gamma}\left(T_{33, \gamma}+b_{\gamma}^{\alpha} T_{\alpha 3}+b_{\gamma}^{\alpha} T_{3 \alpha}\right) \boldsymbol{a}^{3} \otimes \boldsymbol{a}_{\beta}+\epsilon^{\gamma \beta}\left(T_{3 \beta \mid \gamma}+b_{\gamma}^{\alpha} T_{\alpha \beta}\right) \boldsymbol{a}^{3} \otimes \boldsymbol{a}_{3} .
\end{aligned}
$$

Alternatively, one can use the mixed components $T_{\cdot j}^{i}$ and write $\operatorname{Curl}_{s} \boldsymbol{T}$ in the tensor basis $\left\{\boldsymbol{a}_{i} \otimes \boldsymbol{a}_{j}\right\}$

$$
\begin{gathered}
\operatorname{Curl}_{s} \boldsymbol{T}=\epsilon^{\beta \gamma}\left(T_{\cdot 3 \mid \gamma}^{\alpha}+b_{\gamma}^{\sigma} T_{\cdot{ }_{\sigma}}^{\alpha}-b_{\gamma}^{\alpha} T_{\cdot 3}^{3}\right) \boldsymbol{a}_{\alpha} \otimes \boldsymbol{a}_{\beta}+\epsilon^{\gamma \beta}\left(T_{\cdot \beta \mid \gamma}^{\alpha}-b_{\gamma}^{\alpha} T_{\cdot \beta}^{3}\right) \boldsymbol{a}_{\alpha} \otimes \boldsymbol{a}_{3} \\
+\epsilon^{\beta \gamma}\left(T_{\cdot 3, \gamma}^{3}+b_{\alpha \gamma} T_{\cdot 3}^{\alpha}+b_{\gamma}^{\alpha} T_{\cdot \alpha}^{3}\right) \boldsymbol{a}_{3} \otimes \boldsymbol{a}_{\beta}+\epsilon^{\gamma \beta}\left(T_{\cdot \beta \mid \gamma}^{3}+b_{\alpha \gamma} T_{\cdot \beta}^{\alpha}\right) \boldsymbol{a}_{3} \otimes \boldsymbol{a}_{3} .
\end{gathered}
$$

where

$$
\begin{aligned}
& T_{\cdot \beta \mid \gamma}^{\alpha}=T_{\cdot \beta, \gamma}^{\alpha}+\Gamma_{\gamma \sigma}^{\alpha} T_{\cdot \beta}^{\sigma}-\Gamma_{\beta \gamma}^{\sigma} T_{\cdot \sigma}^{\alpha}, \\
& T_{\cdot 3 \mid \gamma}^{\alpha}=T_{\cdot 3, \gamma}^{\alpha}+\Gamma_{\gamma \sigma}^{\alpha} T_{\cdot 3}^{\sigma}, \quad T_{\cdot \beta \mid \gamma}^{3}=T_{\cdot \beta, \gamma}^{3}-\Gamma_{\beta \gamma}^{\sigma} T_{\cdot \sigma}^{3} .
\end{aligned}
$$

Remark 3.2. In order to obtain a formula analogous to $(9)$ and $\sqrt{12}$, $\sqrt{13}$ for $\mathrm{Curl}_{s}$ we write $\boldsymbol{T}$ in the form

$$
\boldsymbol{T}=\boldsymbol{a}^{i} \otimes \boldsymbol{T}_{i}=\boldsymbol{a}_{i} \otimes \boldsymbol{T}^{i} \quad \text { with } \quad \boldsymbol{T}_{i}=T_{i j} \boldsymbol{a}^{j}, \quad \boldsymbol{T}^{i}=T_{\cdot j}^{i} \boldsymbol{a}^{j} .
$$

By differentiating the first equation with respect to $x_{\gamma}$ we get

$$
\begin{aligned}
\boldsymbol{T}_{, \gamma} & =\boldsymbol{a}_{, \gamma}^{i} \otimes \boldsymbol{T}_{i}+\boldsymbol{a}^{i} \otimes \boldsymbol{T}_{i, \gamma}=\left(-\Gamma_{\beta \gamma}^{\alpha} \boldsymbol{a}^{\beta}+b_{\gamma}^{\alpha} \boldsymbol{a}^{3}\right) \otimes \boldsymbol{T}_{\alpha}-b_{\alpha \gamma} \boldsymbol{a}^{\alpha} \otimes \boldsymbol{T}_{3}+\boldsymbol{a}^{i} \otimes \boldsymbol{T}_{i, \gamma} \\
& =\boldsymbol{a}^{\alpha} \otimes\left(\boldsymbol{T}_{\alpha, \gamma}-\Gamma_{\alpha \gamma}^{\beta} \boldsymbol{T}_{\beta}-b_{\alpha \gamma} \boldsymbol{T}_{3}\right)+\boldsymbol{a}^{3} \otimes\left(\boldsymbol{T}_{3, \gamma}+b_{\gamma}^{\alpha} \boldsymbol{T}_{\alpha}\right) .
\end{aligned}
$$

Taking the vector product with $\boldsymbol{a}^{\gamma}$ and using $(34)_{2}$ we find

$$
\mathrm{Curl}_{s} \boldsymbol{T}=-\left[\boldsymbol{a}^{\alpha} \otimes\left(\boldsymbol{T}_{\alpha \mid \gamma}-b_{\alpha \gamma} \boldsymbol{T}_{3}\right)+\boldsymbol{a}^{3} \otimes\left(\boldsymbol{T}_{3, \gamma}+b_{\gamma}^{\alpha} \boldsymbol{T}_{\alpha}\right)\right] \times \boldsymbol{a}^{\gamma},
$$

with $\boldsymbol{T}_{\alpha \mid \gamma}:=\boldsymbol{T}_{\alpha, \gamma}-\Gamma_{\alpha \gamma}^{\beta} \boldsymbol{T}_{\beta}$. Similarly, we obtain

$$
\operatorname{Curl}_{s} \boldsymbol{T}=-\left[\boldsymbol{a}_{\alpha} \otimes\left(\boldsymbol{T}_{\cdot \mid \gamma}^{\alpha}-b_{\gamma}^{\alpha} \boldsymbol{T}^{3}\right)+\boldsymbol{a}_{3} \otimes\left(\boldsymbol{T}_{\cdot, \gamma}^{3}+b_{\alpha \gamma} \boldsymbol{T}^{\alpha}\right)\right] \times \boldsymbol{a}^{\gamma},
$$

with $\boldsymbol{T}_{\cdot{ }_{1 \gamma}}^{\alpha}:=\boldsymbol{T}^{\alpha}{ }_{, \gamma}+\Gamma_{\beta \gamma}^{\alpha} \boldsymbol{T}^{\beta}$. The equations 40 and 41] are the counterpart of the relations 12 and, respectively, 13 in the three-dimensional theory.

\section{The shell dislocation density tensor}

Let us present first the kinematics of Cosserat-type shells, which coincides with the kinematics of the 6-parameter shell model, see [15, 19, 13.

We consider a deformable surface $\omega_{\xi} \subset \mathbb{R}^{3}$ which is identified with the midsurface of the shell in its reference configuration and denote with $\left(\xi_{1}, \xi_{2}, \xi_{3}\right)$ a generic point of the surface. Each material point is assumed to have 6 degrees of freedom ( 3 for translations and 3 for rotations). Thus, the deformation of the Cosserat-type shell is determined by a vectorial map $\boldsymbol{m}_{\xi}$ and the microrotation tensor $\boldsymbol{R}_{\xi}$

$$
\boldsymbol{m}_{\xi}: \omega_{\xi} \rightarrow \omega_{c}, \quad \boldsymbol{R}_{\xi}: \omega_{\xi} \rightarrow \mathrm{SO}(3)
$$


where $\omega_{c}$ denotes the deformed (current) configuration of the surface. We consider a parametric representation $\boldsymbol{y}_{0}$ of the reference configuration $\omega_{\xi}$

$$
\boldsymbol{y}_{0}: \omega \rightarrow \omega_{\xi}, \quad \boldsymbol{y}_{0}\left(x_{1}, x_{2}\right)=\left(\xi_{1}, \xi_{2}, \xi_{3}\right),
$$

where $\omega \subset \mathbb{R}^{2}$ is the bounded variation domain (with Lipschitz boundary) of the parameters $\left(x_{1}, x_{2}\right)$. Using the same notations as in Section 3 , we introduce the base vectors $\boldsymbol{a}_{i}, \boldsymbol{a}^{j}$ and the fundamental tensors $\boldsymbol{a}, \boldsymbol{b}$ for the reference surface $\omega_{\xi}$.

The deformation function $\boldsymbol{m}$ is then defined by the composition

$$
\boldsymbol{m}=\boldsymbol{m}_{\xi} \circ \boldsymbol{y}_{0}: \omega \rightarrow \omega_{c}, \quad \boldsymbol{m}\left(x_{1}, x_{2}\right):=\boldsymbol{m}_{\xi}\left(\boldsymbol{y}_{0}\left(x_{1}, x_{2}\right)\right) .
$$

According to (30), the surface gradient of the deformation has the expression

$$
\operatorname{Grad}_{s} \boldsymbol{m}=\boldsymbol{m}_{, \alpha} \otimes \boldsymbol{a}^{\alpha} .
$$

As in the three-dimensional case (see Section 2) we define the elastic microrotation $\boldsymbol{Q}_{e}$ by the composition

$$
\boldsymbol{Q}_{e}=\boldsymbol{R}_{\xi} \circ \boldsymbol{y}_{0}: \omega \rightarrow \mathrm{SO}(3), \quad \boldsymbol{Q}_{e}\left(x_{1}, x_{2}\right):=\boldsymbol{R}_{\xi}\left(\boldsymbol{y}_{0}\left(x_{1}, x_{2}\right)\right),
$$

the total microrotation $\overline{\boldsymbol{R}}$ by

$$
\overline{\boldsymbol{R}}: \omega \rightarrow \mathrm{SO}(3), \quad \overline{\boldsymbol{R}}\left(x_{1}, x_{2}\right)=\boldsymbol{Q}_{e}\left(x_{1}, x_{2}\right) \boldsymbol{Q}_{0}\left(x_{1}, x_{2}\right),
$$

where $\boldsymbol{Q}_{0}: \omega \rightarrow \mathrm{SO}(3)$ is the initial microrotation, which describes the orientation of points in the reference configuration.

To characterize the orientation and rotation of points in Cosserat-type shells one employs (as in the three-dimensional case) a triad of orthonormal directors attached to each point. We denote by $\boldsymbol{d}_{i}^{0}\left(x_{1}, x_{2}\right)$ the directors in the reference configuration $\omega_{\xi}$ and by $\boldsymbol{d}_{i}\left(x_{1}, x_{2}\right)$ the directors in the deformed configuration $\omega_{c}(i=1,2,3)$. The domain $\omega$ is refered to an orthogonal Cartesian frame $O x_{1} x_{2} x_{3}$ such that $\omega \subset O x_{1} x_{2}$ and let $\boldsymbol{e}_{i}$ be the unit vectors along the coordinate axes $O x_{i}$. Then, the microrotation tensors can be expressed as follows

$$
\boldsymbol{Q}_{e}=\boldsymbol{d}_{i} \otimes \boldsymbol{d}_{i}^{0}, \quad \overline{\boldsymbol{R}}=\boldsymbol{Q}_{e} \boldsymbol{Q}_{0}=\boldsymbol{d}_{i} \otimes \boldsymbol{e}_{i}, \quad \boldsymbol{Q}_{0}=\boldsymbol{d}_{i}^{0} \otimes \boldsymbol{e}_{i} .
$$

Remark 4.1. The initial directors $\boldsymbol{d}_{i}^{0}$ are usually chosen such that

$$
\boldsymbol{d}_{3}^{0}=\boldsymbol{n}_{0}, \quad \boldsymbol{d}_{\alpha}^{0} \cdot \boldsymbol{n}_{0}=0,
$$

i.e. $\boldsymbol{d}_{3}^{0}$ is orthogonal to $\omega_{\xi}$ and $\boldsymbol{d}_{\alpha}^{0}$ belong to the tangent plane. This assumption is not necessary in general, but it will be adopted here since it simplifies many of the subsequent expressions. In the deformed configuration, the director $\boldsymbol{d}_{3}$ is no longer orthogonal to the surface $\omega_{c}$ (the Kirchhof-Love condition is not imposed). One convenient choice of the initial microrotation tensor $\boldsymbol{Q}_{0}=\boldsymbol{d}_{i} \otimes \boldsymbol{e}_{i}$ such that the conditions 44 be satisfied is $\boldsymbol{Q}_{0}=\operatorname{polar}\left(\boldsymbol{a}_{i} \otimes \boldsymbol{e}_{i}\right)$, as it was shown in Remark 10 of 12 .

Let us present next the shell strain and curvature measures. In the 6-parameter shell theory the elastic shell strain tensor $\boldsymbol{E}_{e}$ is defined by [15, 19]

$$
\boldsymbol{E}_{e}=\boldsymbol{Q}_{e}^{T} \operatorname{Grad}_{s} \boldsymbol{m}-\boldsymbol{a} .
$$

To write the components of $\boldsymbol{E}_{e}$ we insert 42 and 43$)_{1}$ into 45

$$
\boldsymbol{E}_{e}=\left(\boldsymbol{d}_{i}^{0} \otimes \boldsymbol{d}_{i}\right)\left(\boldsymbol{m}_{, \alpha} \otimes \boldsymbol{a}^{\alpha}\right)-\boldsymbol{a}_{\alpha} \otimes \boldsymbol{a}^{\alpha}=\left(\boldsymbol{m}_{, \alpha} \cdot \boldsymbol{d}_{i}-\boldsymbol{a}_{\alpha} \cdot \boldsymbol{d}_{i}^{0}\right) \boldsymbol{d}_{i}^{0} \otimes \boldsymbol{a}^{\alpha} .
$$

As a measure of orientation (curvature) change, the elastic shell bending-curvature tensor $\boldsymbol{K}_{e}$ is defined by [15, 19, 13 .

$$
\boldsymbol{K}_{e}=\operatorname{axl}\left(\boldsymbol{Q}_{e}^{T} \boldsymbol{Q}_{e, \alpha}\right) \otimes \boldsymbol{a}^{\alpha}=\boldsymbol{Q}_{0}\left[\operatorname{axl}\left(\overline{\boldsymbol{R}}^{T} \overline{\boldsymbol{R}}_{, \alpha}\right)-\operatorname{axl}\left(\boldsymbol{Q}_{0}^{T} \boldsymbol{Q}_{0, \alpha}\right)\right] .
$$


We remark the analogy to the definition (1) of the wryness tensor $\boldsymbol{\Gamma}$ in the three-dimensional theory. Following the analogy to (2), we employ next the surface curl operator $\mathrm{Curl}_{s}$ defined in Section 3 to introduce the new shell dislocation density tensor $\boldsymbol{D}_{e}$ by

$$
\boldsymbol{D}_{e}=\boldsymbol{Q}_{e}^{T} \mathrm{Curl}_{s} \boldsymbol{Q}_{e} .
$$

In view of relation $(34)_{2}$, we can write this definition in the form

$$
\boldsymbol{D}_{e}=\boldsymbol{Q}_{e}^{T}\left(-\boldsymbol{Q}_{e, \alpha} \times \boldsymbol{a}^{\alpha}\right)=-\left(\boldsymbol{Q}_{e}^{T} \boldsymbol{Q}_{e, \alpha}\right) \times \boldsymbol{a}^{\alpha} .
$$

The tensor $\boldsymbol{D}_{e}$ given by 47 represents an alternative strain measure for orientation (curvature) change in Cosserat-type shells.

In what follows, we want to establish the relationship between the shell bending-curvature tensor $\boldsymbol{K}_{e}$ and the shell dislocation density tensor $\boldsymbol{D}_{e}$. We observe that this relationship is analogous to the corresponding relations $\sqrt{19}, \sqrt{20}$ in the three-dimensional theory. More precisely, in the shell theory it holds

$$
\boldsymbol{D}_{e}=-\boldsymbol{K}_{e}^{T}+\left(\operatorname{tr} \boldsymbol{K}_{e}\right) \mathbf{1}_{3} \quad \text { or equivalently, } \quad \boldsymbol{K}_{e}=-\boldsymbol{D}_{e}^{T}+\frac{1}{2}\left(\operatorname{tr} \boldsymbol{D}_{e}\right) \mathbf{1}_{3} .
$$

To prove 49, we designate the components of the shell bending-curvature tensor by $\boldsymbol{K}_{e}=$ $K_{i \alpha} \boldsymbol{d}_{i}^{0} \otimes \boldsymbol{a}^{\alpha}$ and use $16 r_{3}$ to write

$$
\begin{aligned}
\left(\boldsymbol{Q}_{e}^{T} \boldsymbol{Q}_{e, \alpha}\right) & \otimes \boldsymbol{a}^{\alpha}=-\boldsymbol{\epsilon} \operatorname{axl}\left(\boldsymbol{Q}_{e}^{T} \boldsymbol{Q}_{e, \alpha}\right) \otimes \boldsymbol{a}^{\alpha}=-\boldsymbol{\epsilon} \boldsymbol{K}_{e} \\
& =-\left(e_{i j k} \boldsymbol{d}_{i}^{0} \otimes \boldsymbol{d}_{j}^{0} \otimes \boldsymbol{d}_{k}^{0}\right)\left(K_{s \alpha} \boldsymbol{d}_{s}^{0} \otimes \boldsymbol{a}^{\alpha}\right)=-e_{i j s} K_{s \alpha} \boldsymbol{d}_{i}^{0} \otimes \boldsymbol{d}_{j}^{0} \otimes \boldsymbol{a}^{\alpha},
\end{aligned}
$$

which implies

$$
\boldsymbol{Q}_{e}^{T} \boldsymbol{Q}_{e, \alpha}=-e_{i j s} K_{s \alpha} \boldsymbol{d}_{i}^{0} \otimes \boldsymbol{d}_{j}^{0}
$$

We substitute the last relation into 48 and derive

$$
\begin{aligned}
\boldsymbol{D}_{e} & =\left(e_{i j s} K_{s \alpha} \boldsymbol{d}_{i}^{0} \otimes \boldsymbol{d}_{j}^{0}\right) \times \boldsymbol{a}^{\alpha}=\left(e_{i j s} K_{s \alpha} \boldsymbol{d}_{i}^{0} \otimes \boldsymbol{d}_{j}^{0}\right) \times\left[\left(\boldsymbol{a}^{\alpha} \cdot \boldsymbol{d}_{\beta}^{0}\right) \boldsymbol{d}_{\beta}^{0}\right] \\
& =\left(\boldsymbol{a}^{\alpha} \cdot \boldsymbol{d}_{\beta}^{0}\right)\left[e_{i j s} K_{s \alpha} \boldsymbol{d}_{i}^{0} \otimes\left(\boldsymbol{d}_{j}^{0} \times \boldsymbol{d}_{\beta}^{0}\right)\right]=\left(\boldsymbol{a}^{\alpha} \cdot \boldsymbol{d}_{\beta}^{0}\right)\left[e_{i j s} e_{j \beta m} K_{s \alpha} \boldsymbol{d}_{i}^{0} \otimes \boldsymbol{d}_{m}^{0}\right] \\
& =\left(\boldsymbol{a}^{\alpha} \cdot \boldsymbol{d}_{\beta}^{0}\right)\left[\left(\delta_{i m} \delta_{s \beta}-\delta_{i \beta} \delta_{s m}\right) K_{s \alpha} \boldsymbol{d}_{i}^{0} \otimes \boldsymbol{d}_{m}^{0}\right] \\
& =\left(\boldsymbol{a}^{\alpha} \cdot \boldsymbol{d}_{\beta}^{0}\right)\left[-K_{s \alpha} \boldsymbol{d}_{\beta}^{0} \otimes \boldsymbol{d}_{s}^{0}+K_{\beta \alpha} \boldsymbol{d}_{i}^{0} \otimes \boldsymbol{d}_{i}^{0}\right] \\
& \left.=-K_{i \alpha}\left[\left(\boldsymbol{a}^{\alpha} \cdot \boldsymbol{d}_{\beta}^{0}\right) \boldsymbol{d}_{\beta}^{0}\right] \otimes \boldsymbol{d}_{i}^{0}+K_{\beta \alpha}\left(\boldsymbol{d}_{\beta}^{0} \cdot \boldsymbol{a}^{\alpha}\right) \mathbf{1}_{3}\right] \\
& =-\left(K_{i \alpha} d_{i}^{0} \otimes \boldsymbol{a}^{\alpha}\right)^{T}+\operatorname{tr}\left(K_{i \alpha} d_{i}^{0} \otimes \boldsymbol{a}^{\alpha}\right) \mathbf{1}_{3}=-K_{e}^{T}+\left(\operatorname{tr} K_{e}\right) \mathbf{1}_{3},
\end{aligned}
$$

which shows that $491_{1}$ holds true. Applying the trace operator to equation $491_{1}$ we get $\operatorname{tr} \boldsymbol{K}_{e}=\frac{1}{2} \operatorname{tr} \boldsymbol{D}_{e}$. Inserting this into 49$)_{1}$ we obtain 49$]_{2}$. The proof is complete.

Remark 4.2. As a consequence of relations 49 we deduce the relations between the norms, traces, symmetric and skew-symmetric parts of the two tensors in the forms

$$
\begin{aligned}
& \left\|\boldsymbol{D}_{e}\right\|^{2}=\left\|\boldsymbol{K}_{e}\right\|^{2}+\left(\operatorname{tr} \boldsymbol{K}_{e}\right)^{2}, \quad\left\|\boldsymbol{K}_{e}\right\|^{2}=\left\|\boldsymbol{D}_{e}\right\|^{2}-\frac{1}{4}\left(\operatorname{tr} \boldsymbol{D}_{e}\right)^{2}, \\
& \operatorname{tr} \boldsymbol{D}_{e}=2 \operatorname{tr} \boldsymbol{K}_{e}, \quad \text { skew } \boldsymbol{D}_{e}=\operatorname{skew} \boldsymbol{K}_{e}, \quad \operatorname{dev}_{3} \operatorname{sym} \boldsymbol{D}_{e}=-\operatorname{dev}_{3} \operatorname{sym} \boldsymbol{K}_{e} .
\end{aligned}
$$

Indeed the relations 50 can be easily proved if we apply the operators $t r,\|\cdot\|$, skew, $\operatorname{dev}_{3}$, and sym to the equation $(49)_{1}$. In view of $(50)_{1}$ and $\left(\operatorname{tr} \boldsymbol{K}_{e}\right)^{2} \leq 3\left\|\boldsymbol{K}_{e}\right\|^{2}$, we obtain the estimate

$$
\left\|\boldsymbol{K}_{e}\right\| \leq\left\|\boldsymbol{D}_{e}\right\| \leq 2\left\|\boldsymbol{K}_{e}\right\| .
$$


In what follows, we write the components of the tensors $\boldsymbol{K}_{e}$ and $\boldsymbol{D}_{e}$. To this aim, we use the relations

$$
\begin{aligned}
\boldsymbol{Q}_{e}^{T} \boldsymbol{Q}_{e, \alpha} & =\left(\boldsymbol{d}_{i}^{0} \otimes \boldsymbol{d}_{i}\right)\left(\boldsymbol{d}_{k, \alpha} \otimes \boldsymbol{d}_{k}^{0}+\boldsymbol{d}_{k} \otimes \boldsymbol{d}_{k, \alpha}^{0}\right) \\
& =\left(\boldsymbol{d}_{i} \cdot \boldsymbol{d}_{k, \alpha}\right) \boldsymbol{d}_{i}^{0} \otimes \boldsymbol{d}_{k}^{0}+\boldsymbol{d}_{i}^{0} \otimes \boldsymbol{d}_{i, \alpha}^{0}=\left(\boldsymbol{d}_{i} \cdot \boldsymbol{d}_{k, \alpha}-\boldsymbol{d}_{i}^{0} \cdot \boldsymbol{d}_{k, \alpha}^{0}\right) \boldsymbol{d}_{i}^{0} \otimes \boldsymbol{d}_{k}^{0},
\end{aligned}
$$

which can be proved in the same way as equation $(19)$. We compute the axial vector of the skew-symmetric tensor 52 and find (similar to 20 )

$$
\operatorname{axl}\left(\boldsymbol{Q}_{e}^{T} \boldsymbol{Q}_{e, \alpha}\right)=-\frac{1}{2} e_{i j k}\left(\boldsymbol{d}_{j} \cdot \boldsymbol{d}_{k, \alpha}-\boldsymbol{d}_{j}^{0} \cdot \boldsymbol{d}_{k, \alpha}^{0}\right) \boldsymbol{d}_{i}^{0} .
$$

By virtue of 53 the definition 46 yields

$$
\begin{aligned}
\boldsymbol{K}_{e}= & \frac{1}{2} e_{i j k}\left(\boldsymbol{d}_{j, \alpha} \cdot \boldsymbol{d}_{k}-\boldsymbol{d}_{j, \alpha}^{0} \cdot \boldsymbol{d}_{k}^{0}\right) \boldsymbol{d}_{i}^{0} \otimes \boldsymbol{a}^{\alpha} \\
= & \left(\boldsymbol{d}_{2, \alpha} \cdot \boldsymbol{d}_{3}-\boldsymbol{d}_{2, \alpha}^{0} \cdot \boldsymbol{d}_{3}^{0}\right) \boldsymbol{d}_{1}^{0} \otimes \boldsymbol{a}^{\alpha}+\left(\boldsymbol{d}_{3, \alpha} \cdot \boldsymbol{d}_{1}-\boldsymbol{d}_{3, \alpha}^{0} \cdot \boldsymbol{d}_{1}^{0}\right) \boldsymbol{d}_{2}^{0} \otimes \boldsymbol{a}^{\alpha} \\
& +\left(\boldsymbol{d}_{1, \alpha} \cdot \boldsymbol{d}_{2}-\boldsymbol{d}_{1, \alpha}^{0} \cdot \boldsymbol{d}_{2}^{0}\right) \boldsymbol{d}_{3}^{0} \otimes \boldsymbol{a}^{\alpha},
\end{aligned}
$$

which gives the components $K_{i \alpha}$ of the shell bending-curvature tensor $\boldsymbol{K}_{e}$ in the tensor basis $\left\{\boldsymbol{d}_{i}^{0} \otimes \boldsymbol{a}^{\alpha}\right\}$.

For the components of $\boldsymbol{D}_{e}$, we insert the relation 52 in the equation 48

$$
\boldsymbol{D}_{e}=-\left(\boldsymbol{d}_{i} \cdot \boldsymbol{d}_{k, \alpha}-\boldsymbol{d}_{i}^{0} \cdot \boldsymbol{d}_{k, \alpha}^{0}\right)\left(\boldsymbol{d}_{i}^{0} \otimes \boldsymbol{d}_{k}^{0}\right) \times \boldsymbol{a}^{\alpha} .
$$

Using that $\boldsymbol{d}_{k}^{0} \times \boldsymbol{a}^{\alpha}=\boldsymbol{d}_{k}^{0} \times\left[\left(\boldsymbol{a}^{\alpha} \cdot \boldsymbol{d}_{\beta}^{0}\right) \boldsymbol{d}_{\beta}^{0}\right]=\left(\boldsymbol{a}^{\alpha} \cdot \boldsymbol{d}_{\beta}^{0}\right) e_{k \beta j} \boldsymbol{d}_{j}^{0}$, we obtain

$$
\boldsymbol{D}_{e}=e_{j k \beta}\left(\boldsymbol{d}_{i, \alpha} \cdot \boldsymbol{d}_{k}-\boldsymbol{d}_{i, \alpha}^{0} \cdot \boldsymbol{d}_{k}^{0}\right)\left(\boldsymbol{a}^{\alpha} \cdot \boldsymbol{d}_{\beta}^{0}\right) \boldsymbol{d}_{i}^{0} \otimes \boldsymbol{d}_{j}^{0},
$$

which shows the components of the shell dislocation density tensor in the tensor basis $\left\{\boldsymbol{d}_{i}^{0} \otimes\right.$ $\left.\boldsymbol{d}_{j}^{0}\right\}$.

\section{Remarks and discussion}

Herein we present some other ways to express the shell dislocation density tensor, the shell bending-curvature tensor and discuss their close relationship.

Remark 5.1. It is sometimes useful to express the components of the shell dislocation density tensor $\boldsymbol{D}_{e}$ in the tensor basis $\left\{\boldsymbol{a}^{i} \otimes \boldsymbol{a}_{j}\right\}$. If we multiply the relation $(49)_{2}$ with $\boldsymbol{n}_{0}$ and take into account that $\boldsymbol{K}_{e} \boldsymbol{n}_{0}=\mathbf{0}$, then we find $\mathbf{0}=-\boldsymbol{D}_{e}^{T} \boldsymbol{n}_{0}+\frac{1}{2}\left(\operatorname{tr} \boldsymbol{D}_{e}\right) \boldsymbol{n}_{0}$, which means

$$
\boldsymbol{n}_{0} \boldsymbol{D}_{e}=\frac{1}{2}\left(\operatorname{tr} \boldsymbol{D}_{e}\right) \boldsymbol{n}_{0}
$$

It follows that the components of $\boldsymbol{D}_{e}$ in the directions $\boldsymbol{n}_{0} \otimes \boldsymbol{a}_{\alpha}$ are zero, i.e. $\boldsymbol{D}_{e}$ has the structure

$$
\boldsymbol{D}_{e}=\boldsymbol{D}_{\|}+D_{\alpha 3} \boldsymbol{a}^{\alpha} \otimes \boldsymbol{n}_{0}+\frac{1}{2}\left(\operatorname{tr} \boldsymbol{D}_{e}\right) \boldsymbol{n}_{0} \otimes \boldsymbol{n}_{0},
$$

where $\boldsymbol{D}_{\|}=\boldsymbol{D}_{e} \boldsymbol{a}=D^{\alpha \beta} \boldsymbol{a}_{\alpha} \otimes \boldsymbol{a}_{\beta}=D_{\alpha}{ }^{\beta} \cdot \boldsymbol{a}^{\alpha} \otimes \boldsymbol{a}_{\beta}$ is the planar part of $\boldsymbol{D}_{e}$ (the part in the tangent plane). If we insert (56) into $49{ }_{1}$ and use $\frac{1}{2} \operatorname{tr} \boldsymbol{D}_{e}=\operatorname{tr} \boldsymbol{K}_{e}$, we get

$$
\boldsymbol{D}_{\|}+D_{\alpha 3} \boldsymbol{a}^{\alpha} \otimes \boldsymbol{n}_{0}+\left(\operatorname{tr} \boldsymbol{K}_{e}\right) \boldsymbol{n}_{0} \otimes \boldsymbol{n}_{0}=-K_{i \alpha} \boldsymbol{a}^{\alpha} \otimes \boldsymbol{d}_{i}^{0}+\left(\operatorname{tr} \boldsymbol{K}_{e}\right)\left(\boldsymbol{a}+\boldsymbol{n}_{0} \otimes \boldsymbol{n}_{0}\right),
$$

which implies (in view of (54)) that

$$
D_{\alpha 3}=-K_{3 \alpha}=\boldsymbol{d}_{1} \cdot \boldsymbol{d}_{2, \alpha}-\boldsymbol{d}_{1}^{0} \cdot \boldsymbol{d}_{2, \alpha}^{0} \quad \text { and } \quad \boldsymbol{D}_{\|}=-\left(\boldsymbol{K}_{\|}\right)^{T}+\left(\operatorname{tr} \boldsymbol{K}_{e}\right) \boldsymbol{a},
$$

where $\boldsymbol{K}_{\|}=\boldsymbol{a} \boldsymbol{K}_{e}=K_{\beta \alpha} \boldsymbol{d}_{\beta}^{0} \otimes \boldsymbol{a}^{\alpha}$ is the planar part of $\boldsymbol{K}_{e}$. 
Remark 5.2. We observe that between the planar part $\boldsymbol{D}_{\|}$of $\boldsymbol{D}_{e}$ and the planar part $\boldsymbol{K}_{\|}$of $\boldsymbol{K}_{e}$ there exists a special relationship. The tensor $\boldsymbol{D}_{\|}$is the cofactor of the tensor $\boldsymbol{K}_{\|}$. Let us explain this in more details: for any planar tensor $\boldsymbol{S}=S_{{ }_{\beta}}^{\alpha} \boldsymbol{a}_{\alpha} \otimes \boldsymbol{a}^{\beta}$ we introduce the transformation

$$
T(\boldsymbol{S})=-\boldsymbol{S}^{T}+(\operatorname{tr} \boldsymbol{S}) \boldsymbol{a} .
$$

One can prove that this transformation has the properties

$$
T(T(\boldsymbol{S}))=\boldsymbol{S} \quad \text { and } \quad T(\boldsymbol{S})=-\boldsymbol{c} \boldsymbol{S} \boldsymbol{c},
$$

where the alternator $c$ is defined in (31). Moreover, in view of $59 r_{2}$ and $(31)$ we can write $T(\boldsymbol{S})$ in the tensor basis $\left\{\boldsymbol{a}^{\alpha} \otimes \boldsymbol{a}_{\beta}\right\}$ as follows

$$
T(\boldsymbol{S})=S_{\cdot 2}^{2} \boldsymbol{a}^{1} \otimes \boldsymbol{a}_{1}-S_{\cdot{ }_{1}}^{2} \boldsymbol{a}^{1} \otimes \boldsymbol{a}_{2}-S_{\cdot{ }_{2}}^{1} \boldsymbol{a}^{2} \otimes \boldsymbol{a}_{1}+S_{\cdot{ }_{1}}^{1} \boldsymbol{a}^{2} \otimes \boldsymbol{a}_{2},
$$

which shows that the $2 \times 2$ matrix of the components of $T(\boldsymbol{S})$ in the basis $\left\{\boldsymbol{a}^{\alpha} \otimes \boldsymbol{a}_{\beta}\right\}$ is the cofactor of the matrix of components of $\boldsymbol{S}$ in the basis $\left\{\boldsymbol{a}_{\alpha} \otimes \boldsymbol{a}^{\beta}\right\}$, since

$$
\left(\begin{array}{cc}
S_{\cdot 2}^{2} & -S_{\cdot{ }_{1}}^{2} \\
-S_{\cdot 2}^{1} & S_{\cdot \cdot 1}^{1}
\end{array}\right)=\operatorname{Cof}\left(\begin{array}{cc}
S_{\cdot 1}^{1} & S_{\cdot 2}^{1} \\
S_{\cdot 1}^{2} & S_{\cdot 2}^{2}
\end{array}\right)
$$

If the tensor $\boldsymbol{S}$ is invertible, then from the Cayley-Hamilton relation $\left(\boldsymbol{S}^{T}\right)^{2}-(\operatorname{tr} \boldsymbol{S}) \boldsymbol{S}^{T}+$ $\operatorname{det} \boldsymbol{S}=\mathbf{0}$ and $(58)$ we deduce

$$
T(\boldsymbol{S})=-\boldsymbol{S}^{T}+(\operatorname{tr} \boldsymbol{S}) \boldsymbol{a}=(\operatorname{det} \boldsymbol{S}) \boldsymbol{S}^{-T}=: \operatorname{Cof}(\boldsymbol{S}) .
$$

In our case, for the shell bending-curvature tensor $\boldsymbol{K}_{e}$ we have $\operatorname{tr} \boldsymbol{K}_{e}=\operatorname{tr}\left(\boldsymbol{a} \boldsymbol{K}_{e}\right)=\operatorname{tr}\left(\boldsymbol{K}_{\|}\right)$, in view of (54). Then, the relation $(57)_{2}$ yields

$$
\boldsymbol{D}_{\|}=-\left(\boldsymbol{K}_{\|}\right)^{T}+\left(\operatorname{tr} \boldsymbol{K}_{\|}\right) \boldsymbol{a} .
$$

Using the relations 58 - 60 we see that $\boldsymbol{D}_{\|}$is the image of $\boldsymbol{K}_{\|}$under the transformation $T$, so that it holds

$$
\begin{aligned}
& \boldsymbol{D}_{\|}=T\left(\boldsymbol{K}_{\|}\right)=-\boldsymbol{c}\left(\boldsymbol{K}_{\|}\right) \boldsymbol{c}=\operatorname{Cof}\left(\boldsymbol{K}_{\|}\right), \\
& \boldsymbol{K}_{\|}=T\left(\boldsymbol{D}_{\|}\right)=-\boldsymbol{c}\left(\boldsymbol{D}_{\|}\right) \boldsymbol{c}=\operatorname{Cof}\left(\boldsymbol{D}_{\|}\right) .
\end{aligned}
$$

From 56, 57 we can write

$$
\boldsymbol{D}_{e}=\operatorname{Cof}\left(\boldsymbol{K}_{\|}\right)-K_{3 \alpha} \boldsymbol{a}^{\alpha} \otimes \boldsymbol{n}_{0}+\left(\operatorname{tr} \boldsymbol{K}_{\|}\right) \boldsymbol{n}_{0} \otimes \boldsymbol{n}_{0},
$$

which expresses once again the close relationship between the shell dislocation density tensor $\boldsymbol{D}_{e}$ and the shell bending-curvature tensor $\boldsymbol{K}_{e}$.

Remark 5.3. The shell bending-curvature tensor $\boldsymbol{K}_{e}$ can also be expressed in terms of the directors $\boldsymbol{d}_{i}$. In this respect, an analogous relation to the formula 28 for the wryness tensor (see Remark 2.5 holds

$$
\boldsymbol{K}_{e}=\frac{1}{2}\left[\boldsymbol{Q}_{e}^{T}\left(\boldsymbol{d}_{i} \times \boldsymbol{d}_{i, \alpha}\right)-\boldsymbol{d}_{i}^{0} \times \boldsymbol{d}_{i, \alpha}^{0}\right] \otimes \boldsymbol{a}^{\alpha} .
$$

To prove 63 , we write the two terms in the brackets in the following form

$$
\begin{aligned}
\boldsymbol{Q}_{e}^{T}\left(\boldsymbol{d}_{i} \times \boldsymbol{d}_{i, \alpha}\right) & =\left(\boldsymbol{d}_{k}^{0} \otimes \boldsymbol{d}_{k}\right)\left(\boldsymbol{d}_{i} \times \boldsymbol{d}_{i, \alpha}\right)=\left[\boldsymbol{d}_{k} \cdot\left(\boldsymbol{d}_{i} \times \boldsymbol{d}_{i, \alpha}\right)\right] \boldsymbol{d}_{k}^{0} \\
& =\left[\boldsymbol{d}_{i, \alpha} \cdot\left(\boldsymbol{d}_{k} \times \boldsymbol{d}_{i}\right)\right] \boldsymbol{d}_{k}^{0}=e_{k i j}\left(\boldsymbol{d}_{i, \alpha} \cdot \boldsymbol{d}_{j}\right) \boldsymbol{d}_{k}^{0}
\end{aligned}
$$

and similarly

$$
\boldsymbol{d}_{i}^{0} \times \boldsymbol{d}_{i, \alpha}^{0}=\left[\boldsymbol{d}_{k}^{0} \cdot\left(\boldsymbol{d}_{i}^{0} \times \boldsymbol{d}_{i, \alpha}^{0}\right)\right] \boldsymbol{d}_{k}^{0}=\left[\boldsymbol{d}_{i, \alpha}^{0} \cdot\left(\boldsymbol{d}_{k}^{0} \times \boldsymbol{d}_{i}^{0}\right)\right] \boldsymbol{d}_{k}^{0}=e_{k i j}\left(\boldsymbol{d}_{i, \alpha}^{0} \cdot \boldsymbol{d}_{j}^{0}\right) \boldsymbol{d}_{k}^{0} .
$$


Inserting the last two relations into $(63)$ we obtain

$$
\boldsymbol{K}_{e}=\frac{1}{2} e_{i j k}\left[\left(\boldsymbol{d}_{j, \alpha} \cdot \boldsymbol{d}_{k}\right) \boldsymbol{d}_{i}^{0}-\left(\boldsymbol{d}_{j, \alpha}^{0} \cdot \boldsymbol{d}_{k}^{0}\right) \boldsymbol{d}_{i}^{0}\right] \otimes \boldsymbol{a}^{\alpha},
$$

which holds true, by virtue of (54). Thus, (63) is proved.

We can put the relation (63) in the form

$$
\begin{aligned}
& \boldsymbol{K}_{e}=\boldsymbol{Q}_{e}^{T} \boldsymbol{\omega} \quad \text { where we define } \\
& \boldsymbol{\omega}=\boldsymbol{\omega}_{\alpha} \otimes \boldsymbol{a}^{\alpha} \quad \text { with } \quad \boldsymbol{\omega}_{\alpha}=\frac{1}{2}\left[\boldsymbol{d}_{i} \times \boldsymbol{d}_{i, \alpha}-\boldsymbol{Q}_{e}\left(\boldsymbol{d}_{i}^{0} \times \boldsymbol{d}_{i, \alpha}^{0}\right)\right] .
\end{aligned}
$$

If we compare the relations 64 and the definition 46 , we derive

$$
\boldsymbol{\omega}_{\alpha}=\boldsymbol{Q}_{e} \operatorname{axl}\left(\boldsymbol{Q}_{e}^{T} \boldsymbol{Q}_{e, \alpha}\right)=\operatorname{axl}\left(\boldsymbol{Q}_{e, \alpha} \boldsymbol{Q}_{e}^{T}\right) .
$$

Then, from 16 we deduce $\boldsymbol{Q}_{e, \alpha} \boldsymbol{Q}_{e}^{T}=\boldsymbol{\omega}_{\alpha} \times \mathbf{1}_{3}$ and by multiplication with $\boldsymbol{Q}_{e}$ we find

$$
\boldsymbol{Q}_{e, \alpha}=\boldsymbol{\omega}_{\alpha} \times \boldsymbol{Q}_{e}, \quad \alpha=1,2 .
$$

Thus, the equations $(64), 65$ can be employed for an alternative definition of the shell bending-curvature tensor, namely

$$
\boldsymbol{K}_{e}=\boldsymbol{Q}_{e}^{T} \boldsymbol{\omega}, \quad \text { where } \quad \boldsymbol{\omega}=\boldsymbol{\omega}_{\alpha} \otimes \boldsymbol{a}^{\alpha} \quad \text { and } \quad \boldsymbol{Q}_{e, \alpha}=\boldsymbol{\omega}_{\alpha} \times \boldsymbol{Q}_{e} .
$$

This is the counterpart of the relations (24), 25) for the wryness tensor in the threedimensional theory of Cosserat continua. The relations (67) were used to define the corresponding shell bending-curvature tensor, e.g., in [7] 35].

Remark 5.4. As shown by relations (3) for the three-dimensional case, one can introduce the elastically stored shell energy density $W$ as a function of the shell strain tensor and the shell dislocation density tensor

$$
W=W\left(\boldsymbol{E}_{e}, \boldsymbol{D}_{e}\right) .
$$

If 68 is assumed to be a quadratic convex and coercive function, then the existence of solutions to the minimization problem of the total energy functional for Cosserat shells can be proved in a similar manner as in Theorem 6 of 12. In the proof, one should employ decisively the estimate (51) and the expressions of the shell dislocation density tensor $\boldsymbol{D}_{e}$ established in the previous sections.

\section{References}

[1] H. Altenbach. An alternative determination of transverse shear stiffnesses for sandwich and laminated plates. Int. J. Solids Struct., 37:3503-3520, 2000.

[2] H. Altenbach and V.A. Eremeyev. Direct approach-based analysis of plates composed of functionally graded materials. Arch. Appl. Mech., 78:775-794, 2008.

[3] H. Altenbach and V.A. Eremeyev. On the bending of viscoelastic plates made of polymer foams. Acta Mech., 204:137-154, 2009.

[4] H. Altenbach and V.A. Eremeyev. On the effective stiffness of plates made of hyperelastic materials with initial stresses. Int. J. Non-Lin. Mech., 45:976-981, 2010.

[5] H. Altenbach and P.A. Zhilin. Eine nichtlineare Theorie dünner Dreischichtschalen und ihre Anwendung auf die Stabilitätsuntersuchung eines dreischichtigen Streifens. Technische Mechanik, 3:23-30, 1982. 
[6] H. Altenbach and P.A. Zhilin. A general theory of elastic simple shells (in russian). Uspekhi Mekhaniki, 11:107-148, 1988.

[7] H. Altenbach and P.A. Zhilin. The theory of simple elastic shells. In R. Kienzler, H. Altenbach, and I. Ott, editors, Theories of Plates and Shells. Critical Review and New Applications, Euromech Colloquium 444, pages 1-12. Springer, Heidelberg, 2004.

[8] J. Altenbach, H. Altenbach, and V.A. Eremeyev. On generalized Cosserat-type theories of plates and shells: a short review and bibliography. Arch. Appl. Mech., 80:73-92, 2010.

[9] G. Backus, R. Parker, and C. Constable. Foundations of Geomagnetism. Cambridge University Press, Cambridge, 1996.

[10] M. Bîrsan and H. Altenbach. A mathematical study of the linear theory for orthotropic elastic simple shells. Math. Methods Appl. Sci., 33:1399-1413, 2010.

[11] M. Bîrsan and H. Altenbach. Analysis of the deformation of multi-layered orthotropic cylindrical elastic shells using the direct approach. In H. Altenbach and V.A. Eremeyev, editors, Shell-like Structures: Non-classical Theories and Applications, pages 29-52. Springer-Verlag, Berlin Heidelberg, 2011.

[12] M. Bîrsan and P. Neff. Existence of minimizers in the geometrically non-linear 6parameter resultant shell theory with drilling rotations. Math. Mech. Solids, 19(4):376$397,2014$.

[13] M. Bîrsan and P. Neff. Shells without drilling rotations: A representation theorem in the framework of the geometrically nonlinear 6-parameter resultant shell theory. Int. J. Engng. Sci., 80:32-42, 2014.

[14] M. Bîrsan, T. Sadowski, and D. Pietras. Thermoelastic deformations of cylindrical multi-layered shells using a direct approach. J. Thermal Stresses, 36:749-789, 2013.

[15] J. Chróścielewski, J. Makowski, and W. Pietraszkiewicz. Statics and Dynamics of Multifold Shells: Nonlinear Theory and Finite Element Method (in Polish). Wydawnictwo IPPT PAN, Warsaw, 2004.

[16] E. Cosserat and F. Cosserat. Théorie des corps déformables. Hermann et Fils (reprint 2009), Paris, 1909.

[17] V.A. Eremeyev, L.P. Lebedev, and H. Altenbach. Foundations of Micropolar Mechanics. Springer, Heidelberg - New York - Dordrecht - London, 2013.

[18] V.A. Eremeyev and W. Pietraszkiewicz. The nonlinear theory of elastic shells with phase transitions. J. Elasticity, 74:67-86, 2004.

[19] V.A. Eremeyev and W. Pietraszkiewicz. Local symmetry group in the general theory of elastic shells. J. Elasticity, 85:125-152, 2006.

[20] I.D. Ghiba, P. Neff, A. Madeo, L. Placidi, and G. Rosi. The relaxed linear micromorphic continuum: Existence, uniqueness and continuous dependence in dynamics. Math. Mech. Solids, 20:1171-1197, 2015.

[21] M.E. Gurtin. An Introduction to Continuum Mechanics., volume 158 of Mathematics in Science and Engineering. Academic Press, London, 1. edition, 1981.

[22] J. Lankeit, P. Neff, and F. Osterbrink. Integrability conditions between the first and second cosserat deformation tensor in geometrically nonlinear micropolar models and existence of minimizers. ArXiv:1504.08003, 2016. 
[23] A. Libai and J.G. Simmonds. The Nonlinear Theory of Elastic Shells. Cambridge University Press, Cambridge, 2nd edition, 1998.

[24] A. Madeo, P. Neff, I.D. Ghiba, L. Placidi, and G. Rosi. Wave propagation in relaxed linear micromorphic continua: modelling metamaterials with frequency band gaps. Cont. Mech. Therm., 27:551-570, 2015.

[25] A. Mielke and S. Müller. Lower semi-continuity and existence of minimizers in incremental finite-strain elastoplasticity. Z. Angew. Math. Mech., 86:233-250, 2006.

[26] P. Neff, M. Bîrsan, and F. Osterbrink. Existence theorem for geometrically nonlinear Cosserat micropolar model under uniform convexity requirements. J. Elasticity, 121:119-141, 2015.

[27] P. Neff, I.D. Ghiba, A. Madeo, L. Placidi, and G. Rosi. A unifying perspective: the relaxed linear micromorphic continua. Cont. Mech. Therm., 26:639-681, 2014.

[28] P. Neff and I. Münch. Curl bounds Grad on SO(3). ESAIM: Control, Optimisation and Calculus of Variations, 14:148-159, 2008.

[29] J. Nye. Some geometrical relations in dislocated crystals. Acta Metall., 1:153-162, 1953.

[30] W. Pietraszkiewicz and V.A. Eremeyev. On natural strain measures of the non-linear micropolar continuum. Int. J. Solids Struct., 46:774-787, 2009.

[31] T. Sadowski, M. Bîrsan, and D. Pietras. Multilayered and FGM structural elements under mechanical and thermal loads. Part I: Comparison of finite elements and analytical models. Archives of Civil and Mechanical Engineering, 15:1180-1192, 2015.

[32] B. Svendsen. Continuum thermodynamic models for crystal plasticity including the effects of geometrically necessary dislocations. J. Mech. Phys. Solids, 50(25):1297-1329, 2002.

[33] J. Tambača and I. Velčić. Existence theorem for nonlinear micropolar elasticity. ESAIM: Control, Optimisation and Calculus of Variations., 16:92-110, 2010.

[34] P.A. Zhilin. Mechanics of deformable directed surfaces. Int. J. Solids Struct., 12:635648, 1976.

[35] P.A. Zhilin. Applied Mechanics - Foundations of Shell Theory (in Russian). State Polytechnical University Publisher, Sankt Petersburg, 2006. 